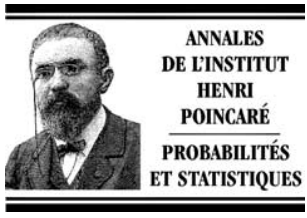

www.elsevier.com/locate/anihpb

\title{
Reversible distributions of multi-allelic Gillespie-Sato diffusion models
}

\author{
Kenji Handa ${ }^{1}$ \\ Department of Mathematics, Saga University, Saga 840-8502, Japan \\ Received 23 August 2002; received in revised form 14 August 2003; accepted 13 January 2004 \\ Available online 12 May 2004 \\ Dedicated to Professor Tokuzo Shiga on the occasion of his 60th birthday
}

\begin{abstract}
We consider multi-allelic Gillespie-Sato diffusion models in population genetics. The case where they have reversible distributions is completely determined in terms of mutation rates and selection intensity. In such cases we give an explicit expression of the reversible distributions, which turn out to be mutually absolutely continuous with respect to some Dirichlet distributions.

(c) 2004 Elsevier SAS. All rights reserved.
\end{abstract}

\section{Résumé}

On considère les modèles de diffusion multi-alleles de Gillepsie-Sato, en génétique des populations. Le cas réversible est complètement déterminé en termes de taux de mutation et d'intensité de sélection. On obtient une expression explicite des distributions réversibles qui se trouvent être absolument continues par rapport à certaines distributions de Dirichlet.

(c) 2004 Elsevier SAS. All rights reserved.

MSC: 60J60; 92D10

Keywords: Gillespie-Sato diffusion models; Wright-Fisher diffusion models; Reversibility; Quasi-invariance; Dirichlet distributions

\section{Introduction and the main result}

In population genetics theory, stochastic methods such as diffusion approximations had been exploited extensively, yielding rich results which are of interest from both genetical and mathematical view points. Among a number of quantities associated with the diffusion models, it is of particular importance to study their stationary distributions. In general, it is quite difficult to give them in explicit way, and stationary distributions which had been found explicitly are usually shown to exhibit a stronger property called reversibility, i.e., at stationarity the process has the same distribution as its time reversal.

E-mail address: handa@ms.saga-u.ac.jp (K. Handa).

1 Partially supported by The Sumitomo Foundation. 
Besides analytical importance of this property, we should mention about a special role of reversibility in the context of population genetics theory. For this purpose, it seems best to cite an account given by Ewens from [5, p. 87] concerning the prospective and retrospective aspects of the processes. "For reversible processes these two aspects have many properties in common, and information about the prospective behavior normally yields almost immediately useful information about the retrospective behavior". See also e.g. [28,29,15,20,17] and $[4, \S 8]$ for various applications of reversibility or time reversal in population genetics models.

In this paper we discuss multi-allelic Gillespie-Sato diffusion models (hereafter G-S diffusions), which were introduced heuristically by Gillespie [7] in a di-allelic case and rigorously derived by Sato [19] in a multi-allelic case. In fact, they did not take effect of mutation into consideration, and what we actually study here are diffusion approximations obtained by Shiga [22,23]. (See also [24] for further development.) He proved not only the wellposedness of the processes in a countably infinite-allelic case but also derived certain measure-valued diffusion processes in a continuum limit of the space of alleles. However, our attempt will be made only for finitely-manyallelic cases because of technical difficulties, and we try to identify the case where the multi-allelic G-S diffusions have stationary reversible distributions, and to find explicit expressions of them.

According to [22], diffusion processes we will be concerned with are described as follows. Let $d$ be an integer greater than 1 and $\mathbf{R}_{+}$be the set of positive numbers. Suppose that $\beta_{1}, \ldots, \beta_{d} \in \mathbf{R}_{+}$and $\gamma_{1}, \ldots, \gamma_{d} \in \mathbf{R}$ are given. We also need a $d \times d$-matrix $\left(\lambda_{i j}\right)$ such that $\lambda_{i j} \geqslant 0(i \neq j)$ and $\sum_{j=1}^{d} \lambda_{i j}=0$. The $d$-allele G-S diffusion has state space

$$
K_{d}=\left\{x=\left(x_{1}, \ldots, x_{d-1}\right)^{\prime}: x_{1} \geqslant 0, \ldots, x_{d-1} \geqslant 0, x_{d}:=1-x_{1}-\cdots-x_{d-1} \geqslant 0\right\},
$$

where ' stands for the transpose. It is prescribed by the generator

$$
L=\frac{1}{2} \sum_{i, j=1}^{d-1} a_{i j}(x) \frac{\partial^{2}}{\partial x_{i} \partial x_{j}}+\sum_{i=1}^{d-1} b_{i}(x) \frac{\partial}{\partial x_{i}}
$$

with coefficients

$$
a_{i j}(x)=\delta_{i j} \beta_{i} x_{i}+x_{i} x_{j}\left(\sum_{k=1}^{d} \beta_{k} x_{k}-\beta_{i}-\beta_{j}\right)
$$

and

$$
b_{i}(x)=\sum_{j=1}^{d} \lambda_{j i} x_{j}+x_{i}\left(\gamma_{i}-\sum_{j=1}^{d} \gamma_{j} x_{j}\right),
$$

where $\delta_{i j}$ denotes the Kronecker's delta. Each $\lambda_{i j}$ represents the rate of mutation from the $i$ th allele, say $A_{i}$, to the $j$ th allele $A_{j}$, and $\gamma_{i}$ involves the effect of natural selection. It is convenient to introduce notation of the scalar product on $\mathbf{R}^{d}$

$$
\langle\boldsymbol{\xi}, \boldsymbol{\eta}\rangle=\sum_{i=1}^{d} \xi_{i} \eta_{i}, \quad \boldsymbol{\xi}=\left(\xi_{1}, \ldots, \xi_{d}\right)^{\prime}, \boldsymbol{\eta}=\left(\eta_{1}, \ldots, \eta_{d}\right)^{\prime} \in \mathbf{R}^{d} .
$$

Put $\boldsymbol{\beta}=\left(\beta_{1}, \ldots, \beta_{d}\right)^{\prime}$ and $\boldsymbol{\gamma}=\left(\gamma_{1}, \ldots, \gamma_{d}\right)^{\prime}$. Letting $\bar{x}=\left(x_{1}, \ldots, x_{d-1}, x_{d}\right)^{\prime} \in \mathbf{R}^{d}$ for $x \in K_{d}$, we have rather simple expression of the above coefficients:

$$
\begin{aligned}
& a_{i j}(x)=\delta_{i j} \beta_{i} x_{i}+x_{i} x_{j}\left(\langle\bar{x}, \boldsymbol{\beta}\rangle-\beta_{i}-\beta_{j}\right), \\
& b_{i}(x)=\sum_{j=1}^{d} \lambda_{j i} x_{j}+x_{i}\left(\gamma_{i}-\langle\bar{x}, \boldsymbol{\gamma}\rangle\right) .
\end{aligned}
$$


Let $\mathbf{1}=(1, \ldots, 1)^{\prime} \in \mathbf{R}^{d}$. If $\boldsymbol{\beta}=C \mathbf{1}$ for some $C>0$, then the G-S diffusion is nothing but the Wright-Fisher diffusion model (hereafter W-F diffusion), more precisely, the diffusion approximation for Wright-Fisher models. (See e.g. [5,3].) Genetically speaking, $\beta_{i}$ 's come from the difference among alleles in variances of offspring numbers. More precisely, the diffusion approximations studied in $[7,19,22,23]$ are based on certain multi-type branching processes, in the definition of which the variance of the offspring distribution of allele $A_{i}$ is $\beta_{i}$ plus a term negligible in large population limit. In the absence of mutation, Gillespie [7] discussed effects of variance in offspring numbers on the fitness of a genotype and on the probability of fixation.

In the present paper, mechanism of mutation is necessary for the process to have a nontrivial equilibrium state. Actually, Shiga proved ([21], Theorem 3.2 and Remark 3.1 combined with the main result of [22]), under certain irreducibility condition we will also assume for the mutation rates, that the G-S diffusion has a unique stationary distribution and is ergodic. As mentioned above, main purpose of this paper is to find an explicit expression for the stationary distribution. But we do not intend to consider all of them since, even for the W-F diffusion case, only reversible stationary distributions are known explicitly. So what we have to do first is to identify the case where the G-S diffusion has a reversible distribution and then we shall compute it in the reversible case. Here is the main result of this paper.

Theorem 1.1. Suppose that $\boldsymbol{\beta} \neq C \mathbf{1}$ for any $C>0$ and that $\left(\lambda_{i j}\right)_{1 \leqslant i, j \leqslant d}$ is irreducible in the sense that for every $i$ and $j$ there exist a chain $i_{0}, i_{1}, \ldots, i_{m}$ in $\{1, \ldots, d\}$ such that $i_{0}=i, i_{m}=j$ and $\lambda_{i_{n-1} i_{n}}>0(n=1, \ldots, m)$. Then the G-S diffusion has a reversible distribution if and only if the mutation rates are of uniform type, i.e.,

$$
\lambda_{i j}=\lambda_{k j}\left(=: q_{j} / 2\right), \quad \text { for all } i, k \in\{1, \ldots, d\} \backslash\{j\} \text { and } j \in\{1, \ldots, d\}
$$

and

$$
\boldsymbol{\gamma}=C \boldsymbol{\beta}+C^{\prime} \mathbf{1} \text { for some constants } C \text { and } C^{\prime} .
$$

In the case where both (1.5) and (1.6) are satisfied, the unique stationary (reversible) distribution is given by

$$
\left\langle\bar{x}, \boldsymbol{\beta}^{-1}\right\rangle^{-\left\langle\boldsymbol{q}, \boldsymbol{\beta}^{-1}\right\rangle-2 C-1} \prod_{i=1}^{d} x_{i}^{q_{i} \beta_{i}^{-1}-1} d x_{1} \cdots d x_{d-1} / Z_{\boldsymbol{\beta}, \boldsymbol{q}, C},
$$

where $\boldsymbol{\beta}^{-1}=\left(\beta_{1}^{-1}, \ldots, \beta_{d}^{-1}\right)^{\prime}, \boldsymbol{q}=\left(q_{1}, \ldots, q_{d}\right)^{\prime}$ is given in (1.5), and $Z_{\boldsymbol{\beta}, \boldsymbol{q}, C}$ is a positive finite constant that makes (1.7) a probability distribution on $K_{d}$.

Remarks. (i) We should note difference between the G-S diffusion case and the W-F diffusion case. The latter is covered by a theorem of Li, Shiga and Yao [16]. They proved, under the same irreducibility assumption as above, that reversibility of the W-F diffusion is equivalent to only the condition (1.5). Thus no condition on $\gamma$ is required in this case. In addition, we can assume $\boldsymbol{\beta}=\mathbf{1}$ without loss of generality, and the stationary distribution is known $[30]$ as

$$
e^{2\langle\bar{x}, \boldsymbol{\gamma}\rangle} \prod_{i=1}^{d} x_{i}^{q_{i}-1} d x_{1} \cdots d x_{d-1} / \text { normalization. }
$$

Note also that distributions of this form are recovered from (1.7) by setting $\boldsymbol{\beta}=\mathbf{1}+C^{-1} \boldsymbol{\gamma}$ or $\boldsymbol{\gamma}=C \boldsymbol{\beta}-C \mathbf{1}$, which satisfies (1.6), and then letting $C \rightarrow \infty$.

(ii) Clearly (1.7) generalizes Dirichlet distributions, that correspond to the case where $\beta=C_{1} \mathbf{1}$ for some $C_{1}>0$. Since (1.7) is absolutely continuous with respect to the Dirichlet distribution with parameter $\left(q_{1} \beta_{1}^{-1}, \ldots, q_{d} \beta_{d}^{-1}\right)$ and the density is bounded above and uniformly positive, normalizability in (1.7) is obvious.

(iii) When $C=0$ in (1.7), an explicit expression of the normalization $Z_{\beta, q, 0}$ will be given in Lemma 3.1 below.

(iv) In the case of $d=2,(1.5)$ and (1.6) are always satisfied, and the stationary distribution (1.7) is derived directly by using integration by parts in one dimension. 
Heuristics behind the proof of Theorem 1.1 is as follows. In view of general facts (e.g. [14,18], [13, Chapter V, Theorem 4.6]) on symmetrizability of nondegenerate operators of the form (1.1), it seems natural to guess that reversibility of the process we consider would be equivalent to the condition that the "drift term" is of the form

$$
b(x):=\left(b_{1}(x), \ldots, b_{d-1}(x)\right)^{\prime}=-\frac{1}{2} a(x) \nabla H(x) \text { for some function } H(x),
$$

where $a(x)=\left(a_{i j}(x)\right)_{1 \leqslant i, j \leqslant d-1}$ and $\nabla H(x)=\left(\partial H / \partial x_{1}, \ldots, \partial H / \partial x_{d-1}\right)^{\prime}$. Furthermore, this reduces to symmetries

$$
\sum_{k=1}^{d-1} a_{i k}(x) \frac{\partial b_{j}(x)}{\partial x_{k}}=\sum_{k=1}^{d-1} a_{j k}(x) \frac{\partial b_{i}(x)}{\partial x_{k}}, \quad i, j \in\{1, \ldots, d-1\} .
$$

Regarding (1.9) as a family of identities between polynomials in $x_{1}, \ldots, x_{d-1}$, one arrives at (1.5) and (1.6). Moreover, after multiplying both sides of (1.8) by the inverse matrix $a(x)^{-1}$, which exists whenever $x$ belongs to

$$
K_{d}^{+}:=\left\{x=\left(x_{1}, \ldots, x_{d-1}\right)^{\prime} \in K_{d}: x_{1}>0, \ldots, x_{d-1}>0, x_{d}>0\right\}
$$

(cf. (1.12) below), a function $H$ satisfying (1.8) on $K_{d}^{+}$is found as

$$
H(x)=\sum_{i=1}^{d} q_{i} \beta_{i}^{-1} \log \left(x_{i}^{-1}\right)+\left(\left\langle\boldsymbol{q}, \boldsymbol{\beta}^{-1}\right\rangle+2 C\right) \log \left\langle\bar{x}, \boldsymbol{\beta}^{-1}\right\rangle .
$$

Lastly, the stationary distribution would be simply given by

$$
e^{-H(x)}(\operatorname{det} a(x))^{-1} d x_{1} \cdots d x_{d-1} / \text { normalization. }
$$

Thus, we need to compute $\operatorname{det} a(x)$ and $a(x)^{-1}$. Such calculations are rather lengthy and summarized in Appendix A. In particular, it will be shown that

$$
\operatorname{det} a(x)=\left(\beta_{1} x_{1}\right) \cdots\left(\beta_{d} x_{d}\right)\left\langle\bar{x}, \boldsymbol{\beta}^{-1}\right\rangle .
$$

Verification of (1.8) and (1.11) for the W-F diffusion can be found, for example, in [1, Appendix F].

In actual proof of Theorem 1.1, we take a strategy similar to [11], in which the same kind of problems are solved for a class of measure-valued diffusion processes of Fleming-Viot's type. This class contains the W-F diffusions as finite-dimensional cases. The strategy allows one to avoid problems which would be caused by degeneracy of $a(x)$ on the boundary of $K_{d}$ (in $\mathbf{R}^{d-1}$ ) and is based on a transformation group $\left\{S_{f}: f \in \mathbf{R}^{d-1}\right\}$ on $K_{d}$ with property

$$
\frac{d}{d u} S_{u f} x=a\left(S_{u f} x\right) f, \quad f=\left(f_{1}, \ldots, f_{d-1}\right)^{\prime} \in \mathbf{R}^{d-1}, x \in K_{d}
$$

Technicalities regarding this group are collected in Appendix B. In this context, reversible distributions (if exist) are interpreted as distributions with certain quasi-invariance property (see Theorem 2.1 below), and some measuretheoretic considerations will yield (1.8), a key in the above heuristics. It is worth noting and observed in Appendix B that a $\sigma$-finite measure

$$
(\operatorname{det} a(x))^{-1} d x_{1} \cdots d x_{d-1} \quad \text { on } K_{d}^{+}
$$

which appeared in (1.11) is invariant under $\left\{S_{f}\right\}$, and that such invariant measures are unique up to multiplicative constants. We also emphasize that existence of the transformation group (1.13) crucially relies on a special structure of the diffusion matrix, i.e.,

$$
\left(a(x)^{-1}\right)_{i j}=\frac{\partial^{2} U}{\partial x_{i} \partial x_{j}}, \quad x \in K_{d}^{+}
$$


for some function $U$ on $K_{d}^{+}$. See Corollary A.1 in Appendix A for an explicit form of $U$. It seems that such structure characterizes a class of diffusion models for which appropriate modification of methods in the present paper are available.

The rest of the paper is organized as follows. In the next section, we will prove Theorem 1.1 by means of results shown in Appendices A and B. Such results would be interesting in their own rights and useful in some other situations. As the last part (besides appendices) of this paper, we discuss some aspects of the reversible distributions obtained in Theorem 1.1, especially logarithmic Sobolev inequalities for the reversible G-S diffusions. This kind of inequalities is known as a powerful tool to study ergodic behaviors of the process and asymptotic stability of the equilibrium distribution. (See e.g. [8] for general accounts and various examples.) In our case these are shown to hold as a direct consequence of logarithmic Sobolev inequalities for the reversible W-F diffusions proved by Stannat [25] combined with remark (ii) after Theorem 1.1.

\section{Quasi-invariance and reversibility}

As for the scalar product on $\mathbf{R}^{d-1}$, we use notation

$$
(f, g)=\sum_{i=1}^{d-1} f_{i} g_{i}, \quad f=\left(f_{1}, \ldots, f_{d-1}\right)^{\prime}, g=\left(g_{1}, \ldots, g_{d-1}\right)^{\prime} \in \mathbf{R}^{d-1} .
$$

Set also $\|f\|_{1}=\left|f_{1}\right|+\cdots+\left|f_{d-1}\right|$. As mentioned in the previous section, one of main tools in this section is the transformation group $\left\{S_{f}: f \in \mathbf{R}^{d-1}\right\}$ on $K_{d}$ constructed in Appendix B. For each $f \in \mathbf{R}^{d-1}$, the image of $x \in K_{d}$ by $S_{f}$ is denoted as

$$
S_{f} x=\left(\left(S_{f} x\right)_{1}, \ldots,\left(S_{f} x\right)_{d-1}\right)^{\prime} \in K_{d} .
$$

Properties of $\left\{S_{f}\right\}$ we employ here are the following. The proofs are found in Appendix B.

(S.1) For each $i \in\{1, \ldots, d\},\left(S_{f} x\right)_{i}=0$ whenever $x_{i}=0$.

(S.2) $S_{0} x=x$ and $S_{f}\left(S_{g} x\right)=S_{f+g} x$. In particular, $S_{-f}=\left(S_{f}\right)^{-1}$.

(S.3) $S_{f} x \in K_{d}^{+}$if and only if $x \in K_{d}^{+}$.

(S.4) For any $x, y \in K_{d}^{+}$, there exists a unique $f \in \mathbf{R}^{d-1}$ such that $y=S_{f} x$.

(S.5) $\frac{\partial\left(S_{f} x\right)_{i}}{\partial f_{j}}=a_{i j}\left(S_{f} x\right), i, j \in\{1, \ldots, d-1\}$.

(S.6) $S_{f} x$ is continuously differentiable in $x$, and

$$
D_{x}\left(S_{f} x\right) a(x)=a\left(S_{f} x\right), \quad \text { where } D_{x}\left(S_{f} x\right)=\left(\frac{\partial\left(S_{f} x\right)_{i}}{\partial x_{j}}\right)_{1 \leqslant i, j \leqslant d-1} .
$$

(S.7) There exist constants $C_{1}$ and $C_{2}$ such that for all $x, y \in K_{d}, f, g \in \mathbf{R}^{d-1}$

$$
\left\|S_{f} x-S_{g} y\right\|_{1} \leqslant\left(\|x-y\|_{1}+C_{1}\|f-g\|_{1}\right) \exp \left(C_{2} \min \left\{\|f\|_{1},\|g\|_{1}\right\}\right) .
$$

(S.8) For any nonnegative Borel function $F$ on $K_{d}^{+}$

$$
\int_{\mathbf{R}^{d-1}} F\left(S_{f} x\right) d f_{1} \cdots d f_{d-1}=\int_{K_{d}^{+}} F(y) m(d y)=\int_{K_{d}^{+}} F\left(S_{g} y\right) m(d y),
$$

where $x \in K_{d}^{+}$and $g \in \mathbf{R}^{d-1}$ are arbitrary and $m(d y)=d y_{1} \cdots d y_{d-1} / \operatorname{det} a(y)$. 
Denote by $\mathcal{M}_{1}\left(K_{d}\right)$ the set of Borel probability measures on $K_{d}$. Given a measurable function $\Lambda$ on $\mathbf{R}^{d-1} \times K_{d}$, we say that a $v \in \mathcal{M}_{1}\left(K_{d}\right)$ is quasi-invariant under $\left\{S_{f}\right\}$ with cocycle $\Lambda$ if for every $f \in \mathbf{R}^{d-1}, v$ and $v \circ S_{f}$ $\left(=v \circ\left(S_{-f}\right)^{-1}\right)$ are mutually absolutely continuous with density given by

$$
\frac{d\left(v \circ S_{f}\right)}{d v}(x)=e^{\Lambda(f, x)}, \quad v \text {-a.s., }
$$

which is equivalent to the condition that

$$
\int_{K_{d}^{+}} F\left(S_{-f} x\right) v(d x)=\int_{K_{d}^{+}} F(x) e^{\Lambda(f, x)} v(d x), \quad f \in \mathbf{R}^{d-1}
$$

holds for any nonnegative Borel function $F$ on $K_{d}^{+}$. In this case, the chain rule, (S.2) and (2.1) together imply that for $f, g \in \mathbf{R}^{d-1}$

$$
\Lambda(f+g, x)=\Lambda\left(f, S_{g} x\right)+\Lambda(g, x), \quad v \text {-a.s. }
$$

This is referred to as cocycle identity. Put $b(x)=\left(b_{1}(x), \ldots, b_{d-1}(x)\right)^{\prime}$.

Theorem 2.1. Let $v \in \mathcal{M}_{1}\left(K_{d}\right)$. Then $v$ is a reversible distribution of the $G-S$ diffusion if and only if $v$ is quasiinvariant under $\left\{S_{f}\right\}$ with cocycle

$$
\Lambda(f, x)=2 \int_{0}^{1}\left(b\left(S_{u f} x\right), f\right) d u
$$

We prepare an equality which plays a key role in proving Theorem 2.1 .

Lemma 2.1. Let $\Lambda$ be given by (2.3). Fix an arbitrary $f \in \mathbf{R}^{d-1}$. For any $G \in C^{1}\left(K_{d}\right)$, define

$$
G_{t}(x)=G\left(S_{-t f} x\right) \exp \left(-\Lambda\left(t f, S_{-t f} x\right)\right), \quad t \in \mathbf{R} .
$$

Then $G_{t} \in C^{1}\left(K_{d}\right)$ and

$$
\frac{d}{d t} G_{t}(x)=-2(b(x), f) G_{t}(x)-\left(a(x) f, \nabla G_{t}(x)\right) .
$$

Proof. Define $V_{t}(x)=\Lambda\left(t f, S_{-t f} x\right)$. We first compute $\nabla G_{t}$ as

$$
\nabla G_{t}(x)=e^{-V_{t}(x)} \nabla\left(G \circ S_{-t f}\right)(x)-G_{t}(x) \nabla V_{t}(x) .
$$

Moreover, by (S.6)

$$
\begin{aligned}
\left(a(x) f, \nabla\left(G \circ S_{-t f}\right)(x)\right) & =\left(a(x) f, D_{x}\left(S_{-t f} x\right)^{\prime} \nabla G\left(S_{-t f} x\right)\right) \\
& =\left(D_{x}\left(S_{-t f} x\right) a(x) f, \nabla G\left(S_{-t f} x\right)\right) \\
& =\left(a\left(S_{-t f} x\right) f, \nabla G\left(S_{-t f} x\right)\right)=-\frac{d}{d t} G\left(S_{-t f} x\right),
\end{aligned}
$$

where (S.5) was used to show the last equality. Observing that

$$
V_{t}(x)=\Lambda\left(t f, S_{-t f} x\right)=2 \int_{0}^{t}\left(b\left(S_{-u f} x\right), f\right) d u,
$$


we see from (S.6) and (2.5) that $G_{t} \in C^{1}\left(K_{d}\right)$. Applying calculations (2.6) to $W(x):=(b(x), f)$ instead of $G(x)$, we get

$$
\begin{aligned}
\left(a(x) f, \nabla V_{t}(x)\right) & =2 \int_{0}^{t}\left(a(x) f, \nabla\left(W \circ S_{-u f}\right)(x)\right) d u \\
& =-2 \int_{0}^{t} \frac{d}{d u} W\left(S_{-u f}\right) d u \\
& =-2\left\{\left(b\left(S_{-t f} x\right), f\right)-(b(x), f)\right\} \\
& =-\frac{d}{d t} V_{t}(x)+2(b(x), f) .
\end{aligned}
$$

Combining (2.5) with (2.6) and (2.8) yields

$$
\begin{aligned}
\left(a(x) f, \nabla G_{t}(x)\right) & =-e^{-V_{t}(x)} \frac{d}{d t} G\left(S_{-t f} x\right)-G_{t}(x)\left\{-\frac{d}{d t} V_{t}(x)+2(b(x), f)\right\} \\
& =-\frac{d}{d t} G_{t}(x)-2(b(x), f) G_{t}(x) .
\end{aligned}
$$

This proves (2.4)

Proof of Theorem 2.1. Firstly, we recall a fundamental fact on reversibility which is implied by Theorem 2.3 of Fukushima and Stroock [6]. Namely, $v \in \mathcal{M}_{1}\left(K_{d}\right)$ is a reversible distribution of the G-S diffusion if and only if the following symmetry holds:

$$
\int(L F)(x) G(x) v(d x)=\int F(x)(L G)(x) v(d x), \quad F, G \in C^{2}\left(K_{d}\right) .
$$

(All integrals are taken over $K_{d}$.) Note that this particularly implies that for any $F \in C^{2}\left(K_{d}\right), \int(L F)(x) v(d x)=0$. Since direct computation shows that

$$
(L F)(x) G(x)+F(x)(L G)(x)-(L(F G))(x)=-(a(x) \nabla F(x), \nabla G(x)),
$$

the above symmetry is equivalent to that

$$
-\int(L F)(x) G(x) v(d x)=\frac{1}{2} \int(a(x) \nabla F(x), \nabla G(x)) v(d x)
$$

holds for any $F, G \in C^{2}\left(K_{d}\right)$. Moreover, by approximation, this can be replaced by the condition that (2.10) holds for any $F \in C^{2}\left(K_{d}\right)$ and $G \in C^{1}\left(K_{d}\right)$.

Once (2.4) has been established, the following argument is standard (cf. [11], proof of Theorem 2.1). We shall describe it, using the same notation as in Lemma 2.1. Taking $G \in C^{1}\left(K_{d}\right)$ and $f \in \mathbf{R}^{d-1}$ arbitrarily and integrating both sides of (2.4) with respect to a $v \in \mathcal{M}_{1}\left(K_{d}\right)$, we have

$$
\int(b(x), f) G_{t}(x) v(d x)+\frac{1}{2} \int\left(a(x) f, \nabla G_{t}(x)\right) v(d x)=-\frac{1}{2} \int \frac{d}{d t} G_{t}(x) v(d x) .
$$

Define $F(x)=(x, f)$ to get a more suggesting form

$$
\int(L F)(x) G_{t}(x) v(d x)+\frac{1}{2} \int\left(a(x) \nabla F(x), \nabla G_{t}(x)\right) v(d x)=-\frac{1}{2} \frac{d}{d t} \int G_{t}(x) v(d x) .
$$


Here $G_{t} \in C^{1}\left(K_{d}\right)$ by Lemma 2.1. If $v$ is a reversible distribution of the G-S diffusion, then the left-hand side of (2.11) vanishes by (2.10) and in particular $\int G_{1}(x) v(d x)=\int G_{0}(x) v(d x)$ or

$$
\int G\left(S_{-f} x\right) \exp \left(-\Lambda\left(f, S_{-f} x\right)\right) v(d x)=\int G(x) v(d x) .
$$

This shows the quasi-invariant property with desired density (2.1). Conversely, if $v$ is quasi-invariant under $\left\{S_{f}\right\}$ with cocycle $\Lambda$, then the right-hand side of (2.11) vanishes. Thus (2.10) holds for any $G \in C^{1}\left(K_{d}\right)$ and $F$ such that $F(x)=(x, f)$ for some $f \in \mathbf{R}^{d-1}$. Furthermore, an inductive argument shows that (2.10) can extend to all functions $F(x)=\left(x, f^{(1)}\right) \cdots\left(x, f^{(n)}\right)$ with $f^{(1)}, \ldots, f^{(n)} \in \mathbf{R}^{d-1}$. Indeed, assuming that both $(F=) F_{1}$ and $F_{2}$ satisfy (2.10) for any $G \in C^{1}\left(K_{d}\right)$, we have by (2.9)

$$
\begin{aligned}
\int( & \left.\left(F_{1} F_{2}\right)\right)(x) G(x) v(d x) \\
= & \int\left(L F_{1}\right)(x) F_{2}(x) G(x) v(d x)+\int F_{1}(x)\left(L F_{2}\right)(x) G(x) v(d x)+\int\left(a(x) \nabla F_{1}(x), \nabla F_{2}(x)\right) G(x) v(d x) \\
= & -\frac{1}{2} \int\left(a(x) \nabla F_{1}(x), \nabla\left(F_{2} G\right)(x)\right) v(d x)-\frac{1}{2} \int\left(a(x) \nabla F_{2}(x), \nabla\left(F_{1} G\right)(x)\right) v(d x) \\
& +\int\left(a(x) \nabla F_{1}(x), \nabla F_{2}(x)\right) G(x) v(d x) \\
= & -\frac{1}{2} \int F_{2}(x)\left(a(x) \nabla F_{1}(x), \nabla G(x)\right) v(d x)-\frac{1}{2} \int F_{1}(x)\left(a(x) \nabla F_{2}(x), \nabla G(x)\right) v(d x) \\
= & -\frac{1}{2} \int\left(a(x) \nabla\left(F_{1} F_{2}\right)(x), \nabla G(x)\right) v(d x) .
\end{aligned}
$$

Therefore, it follows from linearity of (2.10) in $F$ that (2.10) holds true for all polynomials $F(x)$. A suitable approximation procedure (see e.g. Appendix 7 in [3]) concludes that (2.10) are valid for all $F \in C^{2}\left(K_{d}\right)$. This implies reversibility of $v$.

In the next lemma, assuming existence and certain support property, we give a concrete expression of quasiinvariant distributions under $\left\{S_{f}\right\}$ in terms of continuous cocycle $\Lambda$.

Lemma 2.2. (i) Let $\Lambda: \mathbf{R}^{d-1} \times K_{d} \rightarrow \mathbf{R}$ be continuous. Suppose that there exists a $v \in \mathcal{M}_{1}\left(K_{d}\right)$ which is quasiinvariant under $\left\{S_{f}\right\}$ with cocycle $\Lambda$. If $\nu\left(K_{d}^{+}\right)=1$, then

$$
Z(x):=\int_{\mathbf{R}^{d-1}} e^{\Lambda(f, x)} d f_{1} \cdots d f_{d-1}<\infty \quad \text { for all } x \in K_{d}^{+},
$$

and

$$
v(d x)=Z(x)^{-1} m(d x) .
$$

Moreover, for any $f \in \mathbf{R}^{d-1}$ and $x \in K_{d}^{+}$

$$
\Lambda(f, x)=\log Z(x)-\log Z\left(S_{f} x\right) .
$$

(ii) If $H$ is a continuous function on $K_{d}^{+}$such that

$$
Z:=\int_{K_{d}^{+}} e^{-H(x)} m(d x)<\infty
$$


then $v(d x):=e^{-H(x)} m(d x) / Z$ is a unique distribution on $K_{d}^{+}$which is quasi-invariant under $\left\{S_{f}\right\}$ with cocycle $\Lambda(f, x)=H(x)-H\left(S_{f} x\right)$.

Proof. (i) For any nonnegative Borel function $F$ on $K_{d}^{+}$, we have the following equalities by the quasi-invariance supposed.

$$
\int_{K_{d}^{+}} F\left(S_{-f} x\right) v(d x)=\int_{K_{d}^{+}} F(x) e^{\Lambda(f, x)} v(d x), \quad f \in \mathbf{R}^{d-1} .
$$

By integrating both sides with respect to $d f_{1} \cdots d f_{d-1}$ over $\mathbf{R}^{d-1}$, using Fubini's theorem and then (S.8), and noting that $v\left(K_{d}^{+}\right)=1,(2.15)$ becomes

$$
\int_{K_{d}^{+}} F(y) m(d y)=\int_{K_{d}^{+}} F(x) Z(x) v(d x) .
$$

Since the left-hand side is finite for $F(x)=\operatorname{det} a(x), Z(x)<\infty$, v-a.e. Therefore, (2.16) proves (2.13). Especially, the support $\operatorname{supp} v$ of $v$ coincides with $K_{d}$. It is obvious from (S.4) that (2.12) is implied by (2.14). This equality is shown by virtue of cocycle identities (2.2) which hold for all $x \in K_{d}$ and $f, g \in \mathbf{R}^{d-1}$ since $\operatorname{supp} v=K_{d}$ and $\Lambda$ is continuous. Indeed, for any $x \in K_{d}^{+}$and $f \in \mathbf{R}^{d-1}$

$$
Z\left(S_{f} x\right)=\int_{\mathbf{R}^{d-1}} e^{\Lambda\left(g, S_{f} x\right)} d g_{1} \cdots d g_{d-1}=e^{-\Lambda(f, x)} \int_{\mathbf{R}^{d-1}} e^{\Lambda(g+f, x)} d g_{1} \cdots d g_{d-1}=e^{-\Lambda(f, x)} Z(x),
$$

which proves (2.14).

(ii) Let $F$ be an arbitrary nonnegative Borel function on $K_{d}^{+}$. By the last equality of (S.8)

$$
\int_{K_{d}^{+}} F(x) e^{-H(x)} m(d x)=\int_{K_{d}^{+}} F\left(S_{f} x\right) e^{-H\left(S_{f} x\right)} m(d x), \quad f \in \mathbf{R}^{d-1}
$$

or equivalently

$$
\int_{K_{d}^{+}} F(x) v(d x)=\int_{K_{d}^{+}} F\left(S_{f} x\right) e^{H(x)-H\left(S_{f} x\right)} v(d x), \quad f \in \mathbf{R}^{d-1} .
$$

Replacing $F$ by $F \circ S_{-f}$, we get (2.15) with $\Lambda(f, x)=H(x)-H\left(S_{f} x\right)$. This shows the required quasi-invariance property. Since $H$ is continuous, uniqueness follows from the assertion (i).

For $\Lambda$ given by (2.3), we shall verify the condition $v\left(K_{d}^{+}\right)=1$ under the irreducibility assumption of Theorem 1.1. Set $I=\{1, \ldots, d\}$. Define $\boldsymbol{B}=\left(\lambda_{i j}\right)_{i, j \in I}$ and decompose $(b(x), f)$ into

$$
(b(x), f)=\langle\bar{x}, \boldsymbol{B} \hat{f}\rangle+\langle\zeta(x), \hat{f}\rangle, \quad f=\left(f_{1}, \ldots, f_{d-1}\right)^{\prime} \in \mathbf{R}^{d-1},
$$

where $\hat{f}=\left(f_{1}, \ldots, f_{d-1}, 0\right)^{\prime} \in \mathbf{R}^{d}$ and $\zeta(x)=\left(\zeta_{1}(x), \ldots, \zeta_{d}(x)\right)^{\prime}$ is defined by

$$
\zeta_{i}(x)=x_{i}\left(\gamma_{i}-\langle\bar{x}, \boldsymbol{\gamma}\rangle\right), \quad i \in I .
$$

Given $\boldsymbol{\xi}, \boldsymbol{\eta} \in \mathbf{R}^{d}$, define $\xi \boldsymbol{\eta} \in \mathbf{R}^{d}$ by

$$
\boldsymbol{\xi} \eta=\left(\xi_{1} \eta_{1}, \ldots, \xi_{d} \eta_{d}\right)^{\prime} .
$$

Observe that for all $x \in K_{d}$ and $\xi \in \mathbf{R}^{d}$

$$
\langle\zeta(x), \boldsymbol{\xi}\rangle=\langle\bar{x}, \boldsymbol{\gamma} \boldsymbol{\xi}\rangle-\langle\bar{x}, \boldsymbol{\gamma}\rangle\langle\bar{x}, \boldsymbol{\xi}\rangle,
$$


and that

$$
|\langle\zeta(x), \boldsymbol{\xi}\rangle| \leqslant \omega(\boldsymbol{\gamma})\langle\bar{x},|\boldsymbol{\xi}|\rangle,
$$

where $\omega(\boldsymbol{\gamma})=\max \left\{\left|\gamma_{i}-\gamma_{j}\right| ; i, j \in I\right\}$ and $|\xi|=\left(\left|\xi_{1}\right|, \ldots,\left|\xi_{d}\right|\right)^{\prime} \in \mathbf{R}^{d}$. Define $\check{\xi}=\left(\check{\xi}_{1}, \ldots, \check{\xi}_{d-1}\right)^{\prime} \in \mathbf{R}^{d-1}$ by $\check{\xi}_{i}=\xi_{i}-\xi_{d}(i=1, \ldots, d-1)$. It is obvious that

$$
\xi=(\hat{\xi})+\xi_{d} \mathbf{1}, \quad \xi \in \mathbf{R}^{d} .
$$

Lemma 2.3. Suppose that $\boldsymbol{B}=\left(\lambda_{i j}\right)_{i, j \in I}$ is irreducible in the same sense as in Theorem 1.1. If $v \in \mathcal{M}_{1}\left(K_{d}\right)$ is quasi-invariant under $\left\{S_{f}\right\}$ with cocycle $\Lambda$ given by $(2.3)$, then $v\left(K_{d}^{+}\right)=1$.

Proof. Let $f \in \mathbf{R}^{d-1}$ be given. Put $\rho=\omega(\boldsymbol{\gamma})$. Define

$$
F(x)=\exp \left[-2(1+\rho) \int_{0}^{1}\left\langle\overline{S_{u f} x}, \hat{f}\right\rangle d u-2 \int_{0}^{1}\left\langle\zeta\left(S_{u f} x\right), \hat{f}\right\rangle d u\right] .
$$

Then by (2.3) and (2.17)

$$
F(x) \exp \Lambda(f, x)=\exp \left[-2 \int_{0}^{1}\left\langle\overline{S_{u f} x},(1+\rho) \hat{f}-\boldsymbol{B} \hat{f}\right\rangle d u\right] .
$$

So the quasi-invariance (2.1) implies that

$$
\begin{aligned}
& \int_{K_{d}} \exp \left[-2 \int_{0}^{1}\left\langle\overline{S_{u f} x},(1+\rho) \hat{f}-\boldsymbol{B} \hat{f}\right\rangle d u\right] v(d x) \\
& \quad=\int_{K_{d}} \exp \left[-2(1+\rho) \int_{0}^{1}\left\langle\overline{S_{-u f} x}, \hat{f}\right\rangle d u-2 \int_{0}^{1}\left\langle\zeta\left(S_{-u f} x\right), \hat{f}\right\rangle d u\right] v(d x) .
\end{aligned}
$$

Since (2.22) holds true if $\hat{f}$ is replaced by $\hat{f}+C \mathbf{1}=: \xi$ with $C \in \mathbf{R}$ being arbitrary, we have also by (2.20)

$$
\begin{aligned}
& \int_{K_{d}} \exp \left[-2 \int_{0}^{1}\left\langle\overline{S_{u \check{\xi}}},(1+\rho) \xi-\boldsymbol{B} \boldsymbol{\xi}\right\rangle d u\right] v(d x) \\
& \quad=\int_{K_{d}} \exp \left[-2(1+\rho) \int_{0}^{1}\left\langle\overline{S_{-u \check{\xi}}}, \xi\right\rangle d u-2 \int_{0}^{1}\left\langle\zeta\left(S_{-u \check{\xi}} x\right), \xi\right\rangle d u\right] v(d x)
\end{aligned}
$$

for any $\xi \in \mathbf{R}^{d}$. In the case where $\xi_{i} \geqslant 0(i \in I)$, it follows from (2.19) and $\rho=\omega(\boldsymbol{\gamma})$ that

$$
\int_{K_{d}} \exp \left[-2 \int_{0}^{1}\left\langle\overline{S_{u \check{\xi}}},(1+\rho) \boldsymbol{\xi}-\boldsymbol{B} \boldsymbol{\xi}\right\rangle d u\right] v(d x) \leqslant \int_{K_{d}} \exp \left[-2 \int_{0}^{1}\left\langle\overline{S_{-u \check{\xi}} x}, \boldsymbol{\xi}\right\rangle d u\right] v(d x) .
$$

Let $k \in I$ be arbitrary and put $\boldsymbol{\varepsilon}^{(k)}=\left(\delta_{i k}\right)_{i \in I} \in \mathbf{R}^{d}$. For any $c>0$, consider $\boldsymbol{\xi}=c \boldsymbol{\eta}$ with

$$
\boldsymbol{\eta}=\int_{0}^{\infty} e^{-(1+\rho) t} e^{t \boldsymbol{B}} \boldsymbol{\varepsilon}^{(k)} d t \quad \text { and } \quad e^{t \boldsymbol{B}}=\sum_{n=0}^{\infty} \frac{t^{n}}{n !} \boldsymbol{B}^{n}
$$


Then $(1+\rho) \boldsymbol{\eta}-\boldsymbol{B} \boldsymbol{\eta}=\boldsymbol{\varepsilon}^{(k)}$ and hence (2.24) becomes

$$
\int_{K_{d}} \exp \left[-2 c \int_{0}^{1}\left(S_{u c \check{\eta}} x\right)_{k} d u\right] v(d x) \leqslant \int_{K_{d}} \exp \left[-2 c \int_{0}^{1}\left\langle\overline{S_{-u c \check{\eta}} x}, \eta\right\rangle d u\right] v(d x) .
$$

Here the irreducibility of $\boldsymbol{B}$ implies that $\eta_{i}>0$ for each $i \in I$. Therefore the right-hand side of (2.25) tends to 0 as $c \rightarrow \infty$, while it follows from (S.1) that the left-hand side is bounded from below by $v\left(\left\{x \in K_{d}: x_{k}=0\right\}\right)$. Consequently, $x_{k}>0$, v-a.s. Since $k \in I$ is arbitrary, $v\left(K_{d}^{+}\right)=1$ as required.

Before proving Theorem 1.1, we show a simple lemma. Define $\bar{a}(x)=\left(a_{i j}(x)\right)_{i, j \in I}$, where $a_{i j}(x)$ are the same as in (1.3) even for $i=d$ or $j=d$.

Lemma 2.4. Let $\boldsymbol{\xi} \in \mathbf{R}^{d}$ and $g \in \mathbf{R}^{d-1}$. Then

$$
\left.\frac{d}{d t}\left\langle\overline{S_{t g} x}, \boldsymbol{\xi}\right\rangle\right|_{t=0}=\langle\bar{a}(x) \hat{g}, \boldsymbol{\xi}\rangle \text {. }
$$

Proof. Noting that the left-hand side of (2.26) does not change its value after replacement $\xi \mapsto \xi+C \mathbf{1}$, we use (S.5) to get

$$
\left.\frac{d}{d t}\left\langle\overline{S_{t g} x}, \boldsymbol{\xi}\right\rangle\right|_{t=0}=\left.\frac{d}{d t}\left(S_{t g} x, \check{\boldsymbol{\xi}}\right)\right|_{t=0}=(a(x) g, \check{\boldsymbol{\xi}})=(g, a(x) \check{\boldsymbol{\xi}}) .
$$

Hence (2.26) is easily shown by observing that $(a(x) \check{\xi})_{i}=(\bar{a}(x) \xi)_{i}, i \in\{1, \ldots, d-1\}$.

Proof of Theorem 1.1. First suppose that there exists a reversible distribution $v \in \mathcal{M}_{1}\left(K_{d}\right)$ of the G-S diffusion. According to remark (iv) after Theorem 1.1, we assume also that $d \geqslant 3$. By Theorem $2.1 v$ is quasi-invariant under $\left\{S_{f}\right\}$ with cocycle $\Lambda$ given by (2.3). Since we have both irreducibility of $\boldsymbol{B}=\left(\lambda_{i j}\right)$ and continuity of $\Lambda$, Lemmas 2.2(i) and 2.3 together imply that there exists a function $H(x)(:=\log Z(x))$ on $K_{d}^{+}$such that

$$
H(x)-H\left(S_{t f} x\right)=\Lambda(t f, x)=2 \int_{0}^{t}\left(b\left(S_{u f} x\right), f\right) d u, \quad f \in \mathbf{R}^{d-1}
$$

and therefore

$$
(b(x), f)=-\left.\frac{1}{2} \frac{d}{d t} H\left(S_{t f} x\right)\right|_{t=0}, \quad f \in \mathbf{R}^{d-1} .
$$

We claim that $H$ is sufficiently smooth on $K_{d}^{+}$. Fixing $y \in K_{d}^{+}$arbitrarily, consider

$$
G(f):=H\left(S_{f} y\right)=H(y)-2 \int_{0}^{1}\left(b\left(S_{u f} y\right), f\right) d u
$$

as a function of $f \in \mathbf{R}^{d-1}$. Then $G$ is smooth by (2.17) and (S.5). By (S.4) the equation $S_{\Psi(x)} y=x$ defines a map $\Psi: K_{d}^{+} \rightarrow \mathbf{R}^{d-1}$. This is nothing but the inverse of $\mathbf{R}^{d-1} \ni f \mapsto S_{f} y \in K_{d}^{+}$, whose Jacobian matrix is nondegenerate and smooth by (S.5). Hence the inverse function theorem implies smoothness of $\Psi$ and accordingly of the composition $H=G \circ \Psi$.

Consequently (2.27) yields symmetry of the form 


$$
\begin{aligned}
\left.\frac{d}{d t}\left(b\left(S_{t g} x\right), f\right)\right|_{t=0} & =-\left.\frac{1}{2} \frac{\partial^{2}}{\partial t \partial u} H\left(S_{u f+t g} x\right)\right|_{t=0, u=0}=-\left.\frac{1}{2} \frac{\partial^{2}}{\partial t \partial u} H\left(S_{t f+u g} x\right)\right|_{t=0, u=0} \\
& =\left.\frac{d}{d t}\left(b\left(S_{t f} x\right), g\right)\right|_{t=0}, \quad f, g \in \mathbf{R}^{d-1} .
\end{aligned}
$$

Making use of (2.17), (2.18), and (2.26), one can compute the most right-hand side of (2.28) as

$$
\begin{aligned}
\left.\frac{d}{d t}\left(b\left(S_{t f} x\right), g\right)\right|_{t=0} & =\left.\frac{d}{d t}\left\langle\overline{S_{t f} x}, \boldsymbol{B} \hat{g}\right\rangle\right|_{t=0}+\left.\frac{d}{d t}\left\langle\overline{S_{t f} x}, \boldsymbol{\gamma} \hat{g}\right\rangle\right|_{t=0}-\left.\frac{d}{d t}\left(\left\langle\overline{S_{t f} x}, \boldsymbol{\gamma}\right\rangle\left\langle\overline{S_{t f} x}, \hat{g}\right\rangle\right)\right|_{t=0} \\
& =\langle\bar{a}(x) \hat{f}, \boldsymbol{B} \hat{g}\rangle+\langle\bar{a}(x) \hat{f}, \boldsymbol{\gamma} \hat{g}\rangle-\langle\bar{a}(x) \hat{f}, \boldsymbol{\gamma}\rangle\langle\bar{x}, \hat{g}\rangle-\langle\bar{x}, \boldsymbol{\gamma}\rangle\langle\bar{a}(x) \hat{f}, \hat{g}\rangle \\
& =\langle\bar{a}(x) \hat{f}, \boldsymbol{B} \hat{g}\rangle+\langle\bar{a}(x) \hat{f}, \boldsymbol{\gamma}(\hat{g}-\langle\bar{x}, \hat{g}\rangle \mathbf{1})\rangle-\langle\bar{x}, \boldsymbol{\gamma}\rangle\langle\bar{a}(x) \hat{f}, \hat{g}\rangle .
\end{aligned}
$$

Noting that this quantity does not change the value when replacing $f$ and $g$ by $\hat{f}+C_{1} \mathbf{1}=: \boldsymbol{\xi}$ and $\hat{g}+C_{2} \mathbf{1}=: \eta$ respectively and that the last term of (2.29) is symmetric in $f$ and $g$, we see that (2.28) is equivalent to

$$
J_{1}(x)[\boldsymbol{\xi}, \boldsymbol{\eta}]:=\langle\bar{a}(x) \boldsymbol{\xi}, \boldsymbol{B} \boldsymbol{\eta}\rangle+\langle\bar{a}(x) \boldsymbol{\xi}, \boldsymbol{\gamma}(\boldsymbol{\eta}-\langle\bar{x}, \boldsymbol{\eta}\rangle \mathbf{1})\rangle=J_{1}(x)[\boldsymbol{\eta}, \boldsymbol{\xi}], \quad \boldsymbol{\xi}, \boldsymbol{\eta} \in \mathbf{R}^{d} .
$$

Moreover, rewriting the bilinear form $J_{1}(x)$ in terms of $\boldsymbol{\beta}$ by using Lemma A.1 in Appendix A and then removing a symmetric part, we obtain from (2.30)

$$
J_{2}(x)[\xi, \eta]=J_{2}(x)[\eta, \xi], \quad \xi, \eta \in \mathbf{R}^{d},
$$

where

$$
\begin{aligned}
J_{2}(x)[\boldsymbol{\xi}, \boldsymbol{\eta}]= & \langle\bar{x}, \boldsymbol{\beta}(\boldsymbol{\xi}-\langle\bar{x}, \boldsymbol{\xi}\rangle \mathbf{1})(\boldsymbol{B} \boldsymbol{\eta}-\langle\bar{x}, \boldsymbol{B} \boldsymbol{\eta}\rangle \mathbf{1})\rangle-\langle\bar{x}, \boldsymbol{\beta}(\boldsymbol{\xi}-\langle\bar{x}, \boldsymbol{\xi}\rangle \mathbf{1})\rangle\langle\bar{x}, \boldsymbol{\gamma}(\boldsymbol{\eta}-\langle\bar{x}, \boldsymbol{\eta}\rangle \mathbf{1})\rangle \\
= & \langle\bar{x}, \boldsymbol{\beta} \boldsymbol{\xi}(\boldsymbol{B} \boldsymbol{\eta})\rangle-\langle\bar{x}, \boldsymbol{\xi}\rangle\langle\bar{x}, \boldsymbol{\beta}(\boldsymbol{B} \boldsymbol{\eta})\rangle-\langle\bar{x}, \boldsymbol{\beta} \boldsymbol{\xi}\rangle\langle\bar{x}, \boldsymbol{B} \boldsymbol{\eta}\rangle+\langle\bar{x}, \boldsymbol{\beta}\rangle\langle\bar{x}, \boldsymbol{\xi}\rangle\langle\bar{x}, \boldsymbol{B} \boldsymbol{\eta}\rangle \\
& -(\langle\bar{x}, \boldsymbol{\beta} \boldsymbol{\xi}\rangle-\langle\bar{x}, \boldsymbol{\beta}\rangle\langle\bar{x}, \boldsymbol{\xi}\rangle)(\langle\bar{x}, \boldsymbol{\gamma} \boldsymbol{\eta}\rangle-\langle\bar{x}, \boldsymbol{\gamma}\rangle\langle\bar{x}, \boldsymbol{\eta}\rangle) .
\end{aligned}
$$

Let $\boldsymbol{\varepsilon}^{(k)}, k \in I$ be the unit vectors in the proof of Lemma 2.3 and consider equalities

$$
J_{2}(x)\left[\boldsymbol{\varepsilon}^{(i)}, \boldsymbol{\varepsilon}^{(j)}\right]=J_{2}(x)\left[\boldsymbol{\varepsilon}^{(j)}, \boldsymbol{\varepsilon}^{(i)}\right], \quad x \in K_{d}^{+}, i, j \in I .
$$

Clearly

$$
\begin{aligned}
J_{2}(x)\left[\boldsymbol{\varepsilon}^{(i)}, \boldsymbol{\varepsilon}^{(j)}\right]= & x_{i} \beta_{i}\left(\boldsymbol{B} \boldsymbol{\varepsilon}^{(j)}\right)_{i}-x_{i}\left\langle\bar{x}, \boldsymbol{\beta} \cdot \boldsymbol{B} \boldsymbol{\varepsilon}^{(j)}\right\rangle-x_{i} \beta_{i}\left\langle\bar{x}, \boldsymbol{B} \boldsymbol{\varepsilon}^{(j)}\right\rangle \\
& +x_{i}\langle\bar{x}, \boldsymbol{\beta}\rangle\left\langle\bar{x}, \boldsymbol{B} \boldsymbol{\varepsilon}^{(j)}\right\rangle-x_{i}\left(\beta_{i}-\langle\bar{x}, \boldsymbol{\beta}\rangle\right) x_{j}\left(\gamma_{j}-\langle\bar{x}, \boldsymbol{\gamma}\rangle\right) .
\end{aligned}
$$

Given distinct $i, j \in I$, take an arbitrary $k \in I \backslash\{i, j\}$. This is possible because $d \geqslant 3$. We regard (2.33) as identities between polynomials with $d-1$ independent variables $\left\{x_{l}: l \in I, l \neq k\right\}$. Note that

$$
\langle\bar{x}, \boldsymbol{\beta}\rangle=\beta_{k}+\sum_{l \in I \backslash\{k\}} x_{l}\left(\beta_{l}-\beta_{k}\right)
$$

and similarly

$$
\left\langle\bar{x}, \boldsymbol{B} \boldsymbol{\varepsilon}^{(j)}\right\rangle=\left(\boldsymbol{B} \boldsymbol{\varepsilon}^{(j)}\right)_{k}+\sum_{l \in I \backslash\{k\}} x_{l}\left(\left(\boldsymbol{B} \boldsymbol{\varepsilon}^{(j)}\right)_{l}-\left(\boldsymbol{B} \boldsymbol{\varepsilon}^{(j)}\right)_{k}\right)=\lambda_{k j}+\sum_{l \in I \backslash\{k\}} x_{l}\left(\lambda_{l j}-\lambda_{k j}\right) .
$$

Comparing coefficients of a monomial $x_{i}$ in (2.33), we get

$$
\beta_{i}\left(\lambda_{i j}-\lambda_{k j}\right)=0, \quad i \neq j \neq k .
$$

This makes it possible to define $q_{j}=2 \lambda_{i j} \quad(i \neq j)$ for each $j \in I$, and (1.5) has been derived. It follows from the irreducibility of $\left(\lambda_{i j}\right)_{i, j \in I}$ that $\boldsymbol{q}:=\left(q_{1}, \ldots, q_{d}\right)^{\prime} \in \mathbf{R}_{+}^{d}$. 
For each $\xi=\left(\xi_{1}, \ldots, \xi_{d}\right)^{\prime} \in \mathbf{R}^{d}, \boldsymbol{B} \xi$ is now of the form

$$
(\boldsymbol{B} \boldsymbol{\xi})_{i}=\left(\langle\boldsymbol{p}, \boldsymbol{\xi}\rangle-\xi_{i}\right)\langle\boldsymbol{q}, \mathbf{1}\rangle / 2, \quad i \in I,
$$

where $\boldsymbol{p}=\left(p_{1}, \ldots, p_{d}\right)^{\prime} \in K_{d}^{+}$is given by $p_{i}=\langle\boldsymbol{q}, \mathbf{1}\rangle^{-1} q_{i}(i \in I)$. Therefore, the previous expression (2.32) of $J_{2}(x)[\xi, \eta]$ is simplified as follows.

$$
\begin{aligned}
J_{2}(x)[\boldsymbol{\xi}, \boldsymbol{\eta}]= & -\langle\bar{x}, \boldsymbol{\beta}(\boldsymbol{\xi}-\langle\bar{x}, \boldsymbol{\xi}\rangle \mathbf{1})(\boldsymbol{\eta}-\langle\bar{x}, \boldsymbol{\eta}\rangle \mathbf{1})\rangle\langle\boldsymbol{q}, \mathbf{1}\rangle / 2 \\
& -\langle\bar{x}, \boldsymbol{\beta}(\boldsymbol{\xi}-\langle\bar{x}, \boldsymbol{\xi}\rangle \mathbf{1})\rangle\langle\bar{x}, \boldsymbol{\gamma}(\boldsymbol{\eta}-\langle\bar{x}, \boldsymbol{\eta}\rangle \mathbf{1})\rangle .
\end{aligned}
$$

By ignoring the first term in the right-hand side of (2.36) which is symmetric, (2.31) becomes

$$
J_{3}(x)[\boldsymbol{\xi}, \boldsymbol{\eta}]:=\langle\bar{x}, \boldsymbol{\beta}(\boldsymbol{\xi}-\langle\bar{x}, \boldsymbol{\xi}\rangle \mathbf{1})\rangle\langle\bar{x}, \boldsymbol{\gamma}(\boldsymbol{\eta}-\langle\bar{x}, \boldsymbol{\eta}\rangle \mathbf{1})\rangle=J_{3}(x)[\boldsymbol{\eta}, \boldsymbol{\xi}], \quad \xi, \boldsymbol{\eta} \in \mathbf{R}^{d} .
$$

Consider again the special cases:

$$
J_{3}(x)\left[\boldsymbol{\varepsilon}^{(i)}, \boldsymbol{\varepsilon}^{(j)}\right]=J_{3}(x)\left[\boldsymbol{\varepsilon}^{(j)}, \boldsymbol{\varepsilon}^{(i)}\right], \quad x \in K_{d}^{+}, i, j \in I .
$$

By the assumption that $\boldsymbol{\beta} \neq C \mathbf{1}$ for any $C$, there exists a $k \in I$ such that $I_{k}:=\left\{i \in I: \beta_{i} \neq \beta_{k}\right\}$ has at least two distinct elements. We fix such a $k$ and take $i, j \in I \backslash\{k\}$ such that $i \neq j$. Observing that

$$
J_{3}(x)\left[\boldsymbol{\varepsilon}^{(i)}, \boldsymbol{\varepsilon}^{(j)}\right]=x_{i}\left(\beta_{i}-\langle\bar{x}, \boldsymbol{\beta}\rangle\right) x_{j}\left(\gamma_{j}-\langle\bar{x}, \boldsymbol{\gamma}\rangle\right)
$$

and using (2.35), one can rewrite (2.38) as

$$
\begin{aligned}
& x_{i}\left(\beta_{i}-\beta_{k}-\sum_{l \in I \backslash\{k\}} x_{l}\left(\beta_{l}-\beta_{k}\right)\right) x_{j}\left(\gamma_{j}-\gamma_{k}-\sum_{l \in I \backslash\{k\}} x_{l}\left(\gamma_{l}-\gamma_{k}\right)\right) \\
& \quad=x_{i}\left(\beta_{j}-\beta_{k}-\sum_{l \in I \backslash\{k\}} x_{l}\left(\beta_{l}-\beta_{k}\right)\right) x_{j}\left(\gamma_{i}-\gamma_{k}-\sum_{l \in I \backslash\{k\}} x_{l}\left(\gamma_{l}-\gamma_{k}\right)\right),
\end{aligned}
$$

which is also regarded as identities between polynomials with $d-1$ independent variables $\left\{x_{l}: l \in I, l \neq k\right\}$. So by comparing coefficients of $x_{i} x_{j}$, one reduces to

$$
\left(\beta_{i}-\beta_{k}\right)\left(\gamma_{j}-\gamma_{k}\right)=\left(\beta_{j}-\beta_{k}\right)\left(\gamma_{i}-\gamma_{k}\right), \quad i \neq k \neq j .
$$

This implies the existence of a constant $C$ such that

$$
\gamma_{i}-\gamma_{k}=C\left(\beta_{i}-\beta_{k}\right), \quad i \in I_{k} \cup\{k\} .
$$

The restriction $i \in I_{k} \cup\{k\}$ in (2.40) can be removed as follows. If $i \notin I_{k} \cup\{k\}$, then $i \neq k$ and $\beta_{i}=\beta_{k}$. On the other hand, taking $j \in I_{k}$ arbitrarily, we see that $i, j, k$ are mutually distinct and hence by (2.39)

$$
\left(\beta_{j}-\beta_{k}\right)\left(\gamma_{i}-\gamma_{k}\right)=\left(\beta_{i}-\beta_{k}\right)\left(\gamma_{j}-\gamma_{k}\right)=0,
$$

which shows that

$$
\gamma_{i}-\gamma_{k}=0=C\left(\beta_{i}-\beta_{k}\right) .
$$

Consequently

$$
\gamma_{i}-C \beta_{i}=\gamma_{k}-C \beta_{k}, \quad i \in I
$$

Thus $\boldsymbol{\gamma}=C \boldsymbol{\beta}+C^{\prime} \mathbf{1}$ holds with $C^{\prime}=\gamma_{k}-C \beta_{k}$. This proves (1.6).

It remains to show that, under the conditions (1.5) and (1.6), (1.7) is a reversible distribution of the G-S diffusion. (As mentioned in the paragraph above Theorem 1.1, the uniqueness of stationary distribution is due to Shiga.) According to these conditions, $b_{i}(x)$ 's are of the form

$$
b_{i}(x)=\frac{1}{2}\left(q_{i}-\langle\boldsymbol{q}, \mathbf{1}\rangle x_{i}\right)+C x_{i}\left(\beta_{i}-\langle\bar{x}, \boldsymbol{\beta}\rangle\right), \quad i=1, \ldots, d-1 .
$$


By virtue of Theorem 2.1, Lemmas 2.2 and 2.3, it suffices to verify an equality

$$
(b(x), f)=-\left.\frac{1}{2} \frac{d}{d t} H\left(S_{t f} x\right)\right|_{t=0}, \quad x \in K_{d}^{+}, f \in \mathbf{R}^{d-1}
$$

or

$$
b(x)=-\frac{1}{2} a(x) \nabla H(x), \quad x \in K_{d}^{+},
$$

where

$$
H(x)=\sum_{i=1}^{d} q_{i} \beta_{i}^{-1} \log \left(x_{i}^{-1}\right)+\left(\left\langle\boldsymbol{q}, \boldsymbol{\beta}^{-1}\right\rangle+2 C\right) \log \left\langle\bar{x}, \boldsymbol{\beta}^{-1}\right\rangle .
$$

Indeed, $H$ satisfies the assumption of Lemma 2.2(ii), and so $v(d x):=e^{-H(x)} m(d x) / Z$ is quasi-invariant under $\left\{S_{f}\right\}$ with cocycle $H(x)-H\left(S_{f} x\right)$, which equals $\Lambda(f, x)$ given by (2.3) because of (2.42). Therefore, it follows from Theorem 2.1 that $v$ is a reversible distribution of the G-S diffusion, and the coincidence of $v$ with (1.7) is seen from the formula for $\operatorname{det} a(x)$ given in Proposition A.1 of Appendix A.

Since by direct calculation

$$
-\frac{1}{2} \frac{\partial H}{\partial x_{i}}=\frac{q_{i}}{2 \beta_{i} x_{i}}-\frac{q_{d}}{2 \beta_{d} x_{d}}-\frac{\left\langle q, \boldsymbol{\beta}^{-1}\right\rangle+2 C}{2\left\langle\bar{x}, \boldsymbol{\beta}^{-1}\right\rangle}\left(\beta_{i}{ }^{-1}-\beta_{d}{ }^{-1}\right),
$$

(2.43) is verified straightforwardly. (Alternatively, compute $a(x)^{-1} b(x)$ by using (2.41) and (A.6) in Appendix A to deduce the right-hand side of (2.44).) The proof of Theorem 1.1 is completed.

\section{Analytic aspects of reversible distributions}

In this section, we discuss some aspects of reversible distributions obtained in Theorem 1.1, namely

$$
P_{\boldsymbol{\beta}, \boldsymbol{q}, C}(d x):=\left\langle\bar{x}, \boldsymbol{\beta}^{-1}\right\rangle^{-\left\langle\boldsymbol{q}, \boldsymbol{\beta}^{-1}\right\rangle-2 C-1} \prod_{i=1}^{d} x_{i}^{q_{i} \beta_{i}^{-1}-1} d x_{1} \cdots d x_{d-1} / Z_{\boldsymbol{\beta}, \boldsymbol{q}, C}
$$

where $\boldsymbol{\beta}^{-1}=\left(\beta_{1}^{-1}, \ldots, \beta_{d}^{-1}\right)^{\prime}, \boldsymbol{q}=\left(q_{1}, \ldots, q_{d}\right)^{\prime} \in \mathbf{R}_{+}^{d}, C \in \mathbf{R}$, and $Z_{\boldsymbol{\beta}, \boldsymbol{q}, C}$ is the normalization constant. In case of $\boldsymbol{\beta}=\mathbf{1}, P_{\mathbf{1}, \boldsymbol{q}, C}=P_{\mathbf{1}, \boldsymbol{q}, 0}$ coincides with the Dirichlet distribution $D_{\boldsymbol{q}}$ with parameter $\boldsymbol{q}$ :

$$
D_{\boldsymbol{q}}(d x)=\frac{\Gamma(\langle\boldsymbol{q}, \mathbf{1}\rangle)}{\prod_{i=1}^{d} \Gamma\left(q_{i}\right)} \prod_{i=1}^{d} x_{i}^{q_{i}-1} d x_{1} \cdots d x_{d-1},
$$

where $\Gamma(\cdot)$ stands for the gamma function. We see from (3.1) and (3.2) that $P_{\boldsymbol{\beta}, \boldsymbol{q}, C}$ and $D_{\boldsymbol{q} \boldsymbol{\beta}^{-1}}$ are equivalent and that the density function satisfies the following uniform bound

$$
\left|\log \frac{d P_{\boldsymbol{\beta}, \boldsymbol{q}, C}}{d D_{\boldsymbol{q} \boldsymbol{\beta}^{-1}}}(x)\right| \leqslant 2\left|\left\langle\boldsymbol{q}, \boldsymbol{\beta}^{-1}\right\rangle+2 C+1\right| \max _{1 \leqslant i \leqslant d}\left|\log \beta_{i}\right|, \quad x \in K_{d}^{+} .
$$

The integral

$$
Z_{\boldsymbol{\beta}, \boldsymbol{q}, C} \frac{\Gamma\left(\left\langle\boldsymbol{q}, \boldsymbol{\beta}^{-1}\right\rangle\right)}{\prod_{i=1}^{d} \Gamma\left(q_{i} \beta_{i}^{-1}\right)}=\int_{K_{d}^{+}}\left\langle\bar{x}, \boldsymbol{\beta}^{-1}\right\rangle^{-\left\langle\boldsymbol{q}, \boldsymbol{\beta}^{-1}\right\rangle-2 C-1} D_{\boldsymbol{q} \boldsymbol{\beta}^{-1}}(d x)
$$

can be thought of as expectation with respect to a distribution of mean of a Dirichlet process, finite dimensional laws of which are Dirichlet distributions. Such processes have been studied in many contexts. (See [2] and references 
therein.) Among analytic methods available, we employ quasi-invariance of $D_{q}$ to study the right-hand side of (3.4). Note that quasi-invariance of the Dirichlet process follows from that of the corresponding gamma process found in [26] and [27]. See also [10]. We will use the following notation.

$$
\begin{aligned}
& \log \boldsymbol{\alpha}=\left(\log \alpha_{1}, \ldots, \log \alpha_{d}\right)^{\prime}, \quad \boldsymbol{\alpha}=\left(\alpha_{1}, \ldots, \alpha_{d}\right)^{\prime} \in \mathbf{R}_{+}^{d}, \\
& \exp \boldsymbol{\xi}=\left(e^{\xi_{1}}, \ldots, e^{\xi_{d}}\right)^{\prime}, \quad \xi=\left(\xi_{1}, \ldots, \xi_{d}\right)^{\prime} \in \mathbf{R}^{d} .
\end{aligned}
$$

Lemma 3.1. Let $\boldsymbol{\alpha} \in \mathbf{R}_{+}^{d}$ and $\lambda \in \mathbf{R}$. Then

$$
\int_{K_{d}^{+}}\left\langle\bar{x}, \boldsymbol{\alpha}^{-1}\right\rangle^{-\langle\boldsymbol{q}, \mathbf{1}\rangle-\lambda} D_{\boldsymbol{q}}(d x)=e^{\langle\boldsymbol{q}, \log \boldsymbol{\alpha}\rangle} \int_{K_{d}^{+}}\langle\bar{x}, \boldsymbol{\alpha}\rangle^{\lambda} D_{\boldsymbol{q}}(d x) .
$$

In particular, the normalization $Z_{\boldsymbol{\beta}, \boldsymbol{q}, 0}$ for $C=0$ is expressed as

$$
Z_{\boldsymbol{\beta}, \boldsymbol{q}, 0} \frac{\Gamma\left(\left\langle\boldsymbol{q}, \boldsymbol{\beta}^{-1}\right\rangle\right)}{\prod_{i=1}^{d} \Gamma\left(q_{i} \beta_{i}^{-1}\right)}=e^{\left\langle\boldsymbol{q} \boldsymbol{\beta}^{-1}, \log \boldsymbol{\beta}\right\rangle} \int_{K_{d}^{+}}\langle\bar{x}, \boldsymbol{\beta}\rangle D_{\boldsymbol{q} \boldsymbol{\beta}^{-1}}(d x)=e^{\left\langle\boldsymbol{q} \boldsymbol{\beta}^{-1}, \log \boldsymbol{\beta}\right\rangle} \frac{\langle\boldsymbol{q}, \mathbf{1}\rangle}{\left\langle\boldsymbol{q}, \boldsymbol{\beta}^{-1}\right\rangle} .
$$

Proof. We give a self-contained proof by applying Theorem 2.1 to the case where $\boldsymbol{\beta}=\mathbf{1}$ and $b_{i}(x)=\left(q_{i}-\right.$ $\left.\langle\boldsymbol{q}, \mathbf{1}\rangle x_{i}\right) / 2, i \in\{1, \ldots, d-1\}$. In this case, the associated transformations, denoted by $S_{f}^{\mathbf{1}}$, take the form

$$
\left(S_{f}^{\mathbf{1} x}\right)_{i}=\frac{e^{f_{i}} x_{i}}{\sum_{j=1}^{d-1} e^{f_{j}} x_{j}+x_{d}}=\frac{e^{f_{i}} x_{i}}{\langle\bar{x}, \exp \hat{f}\rangle}, \quad i \in\{1, \ldots, d-1\}
$$

(see (A.24) for the proof), and this allows one to compute

$$
\Lambda(f, x):=2 \int_{0}^{1}\left(b\left(S_{u f}^{\mathbf{1}} x\right), f\right) d u=\langle\boldsymbol{q}, \mathbf{1}\rangle(\langle\boldsymbol{p}, \hat{f}\rangle-\log \langle\bar{x}, \exp \hat{f}\rangle),
$$

where $\boldsymbol{p}=\langle\boldsymbol{q}, \mathbf{1}\rangle^{-1} \boldsymbol{q}$. By the last half of Theorem 1.1 and Theorem 2.1 with $\boldsymbol{\beta}=\mathbf{1}$ together $D_{\boldsymbol{q}}=P_{\mathbf{1}, \boldsymbol{q}, 0}$ is quasiinvariant under $\left\{S_{f}^{\mathbf{1}}\right\}$ with cocycle given by (3.8). Hence, putting $\xi=\log \boldsymbol{\alpha} \in \mathbf{R}^{d}$ and defining $\breve{\xi} \in \mathbf{R}^{d-1}$ in the same way as in Section 2, we have

$$
\begin{aligned}
& \int_{K_{d}^{+}}\left\langle\bar{x}, \boldsymbol{\alpha}^{-1}\right\rangle^{-\langle\boldsymbol{q}, \mathbf{1}\rangle-\lambda} D_{\boldsymbol{q}}(d x) \\
& \quad=\int_{K_{d}^{+}}\left\langle\overline{S_{-\breve{\xi}}^{\mathbf{1}} x}, \boldsymbol{\alpha}\right\rangle^{\langle\boldsymbol{q}, \mathbf{1}\rangle+\lambda} D_{\boldsymbol{q}}(d x)=\int_{K_{d}^{+}}\langle\bar{x}, \boldsymbol{\alpha}\rangle^{\langle\boldsymbol{q}, \mathbf{1}\rangle+\lambda} e^{\Lambda(\check{\boldsymbol{\xi}}, x)} D_{\boldsymbol{q}}(d x) \\
& =e^{\langle\boldsymbol{q}, \boldsymbol{\xi}\rangle} \int_{K_{d}^{+}}\langle\bar{x}, \boldsymbol{\alpha}\rangle^{\langle\boldsymbol{q}, \mathbf{1}\rangle+\lambda} \exp \{-\langle\boldsymbol{q}, \mathbf{1}\rangle \log \langle\bar{x}, \exp \boldsymbol{\xi}\rangle\} D_{\boldsymbol{q}}(d x)=e^{\langle\boldsymbol{q}, \log \boldsymbol{\alpha}\rangle} \int_{K_{d}^{+}}\langle\bar{x}, \boldsymbol{\alpha}\rangle^{\lambda} D_{\boldsymbol{q}}(d x),
\end{aligned}
$$

proving (3.5). In the above, the first equality follows from (3.7), while the third equality uses (3.8) and the relation (2.20).

It is easily seen from (3.8) combined with an obvious equality

$$
\left.\frac{d}{d u} \int_{K_{d}^{+}} e^{\Lambda(u f, x)} D_{\boldsymbol{q}}(d x)\right|_{u=0}=\left.\frac{d}{d u} \int_{K_{d}^{+}} D_{\boldsymbol{q}}(d x)\right|_{u=0}=0
$$


holding for each $f \in \mathbf{R}^{d-1}$ that

$$
\int_{K_{d}^{+}}(\langle\boldsymbol{p}, \hat{f}\rangle-\langle\bar{x}, \hat{f}\rangle) D_{\boldsymbol{q}}(d x)=0,
$$

and hence

$$
\int_{K_{d}^{+}}\langle\bar{x}, \boldsymbol{\xi}\rangle D_{\boldsymbol{q}}(d x)=\langle\boldsymbol{p}, \boldsymbol{\xi}\rangle, \quad \boldsymbol{\xi} \in \mathbf{R}^{d} .
$$

So letting $\lambda=1$ in (3.5) yields

$$
\int_{K_{d}^{+}}\left\langle\bar{x}, \boldsymbol{\alpha}^{-1}\right\rangle^{-\langle\boldsymbol{q}, \mathbf{1}\rangle-1} D_{\boldsymbol{q}}(d x)=e^{\langle\boldsymbol{q}, \log \boldsymbol{\alpha}\rangle}\langle\boldsymbol{p}, \boldsymbol{\alpha}\rangle=e^{\langle\boldsymbol{q}, \log \boldsymbol{\alpha}\rangle}\langle\boldsymbol{q}, \boldsymbol{\alpha}\rangle /\langle\boldsymbol{q}, \mathbf{1}\rangle .
$$

Replacing $\boldsymbol{q}$ and $\boldsymbol{\alpha}$ by $\boldsymbol{q} \boldsymbol{\beta}^{-1}$ and $\boldsymbol{\beta}$, respectively, we get the last equality in (3.6). The first equality is shown by combining (3.4) with (3.5). We complete the proof.

Since (3.6) can be rewritten as

$$
Z_{\boldsymbol{\beta}, \boldsymbol{q}, 0}{ }^{-1}=\frac{\Gamma\left(\left\langle\boldsymbol{q}, \boldsymbol{\beta}^{-1}\right\rangle\right)}{\prod_{i=1}^{d} \Gamma\left(q_{i} \beta_{i}^{-1}\right)} \frac{\left\langle\boldsymbol{q}, \boldsymbol{\beta}^{-1}\right\rangle}{\langle\boldsymbol{q}, \mathbf{1}\rangle} \prod_{i=1}^{d}\left(\frac{1}{\beta_{i}}\right)^{q_{i} \beta_{i}^{-1}},
$$

we have a more explicit form of $P_{\boldsymbol{\beta}, \boldsymbol{q}, 0}(d x)$ :

$$
\frac{\Gamma\left(\left\langle\boldsymbol{q}, \boldsymbol{\beta}^{-1}\right\rangle\right)}{\prod_{i=1}^{d} \Gamma\left(q_{i} \beta_{i}^{-1}\right)} \frac{\left\langle\boldsymbol{q}, \boldsymbol{\beta}^{-1}\right\rangle}{\langle\boldsymbol{q}, \mathbf{1}\rangle\left\langle\bar{x}, \boldsymbol{\beta}^{-1}\right\rangle}\left\langle\bar{x}, \boldsymbol{\beta}^{-1}\right\rangle^{-\left\langle\boldsymbol{q}, \boldsymbol{\beta}^{-1}\right\rangle} \prod_{i=1}^{d}\left(\frac{x_{i}}{\beta_{i}}\right)^{q_{i} \beta_{i}-1} \frac{d x_{1} \cdots d x_{d-1}}{x_{1} \cdots x_{d-1} x_{d}} .
$$

As the final topic, we discuss logarithmic Sobolev inequalities for reversible G-S diffusions. In view of Theorem 1.1, this means that we restrict ourselves to the case where

$$
b_{i}(x)=\frac{1}{2}\left(q_{i}-\langle\boldsymbol{q}, \mathbf{1}\rangle x_{i}\right)+C x_{i}\left(\beta_{i}-\langle\bar{x}, \boldsymbol{\beta}\rangle\right), \quad i \in\{1, \ldots, d-1\} .
$$

In addition, $P_{\beta, q, C}$ is the corresponding reversible distribution. In the W-F diffusions case (i.e., the case where $\boldsymbol{\beta}=$ const.1), this kind of inequalities was shown to hold by Stannat [25]. In order to describe such inequalities, we need a bilinear form

$$
\mathcal{E}_{\boldsymbol{q}, C}^{\beta}(F, G)=\frac{1}{2} \int_{K_{d}^{+}}\left(a^{\beta}(x) \nabla F(x), \nabla G(x)\right) P_{\boldsymbol{\beta}, \boldsymbol{q}, C}(d x), \quad F, G \in C^{\infty}\left(K_{d}\right),
$$

where $C^{\infty}\left(K_{d}\right)=\left\{F \in C\left(K_{d}\right): F(x)=G(x)\left(\forall x \in K_{d}\right)\right.$ for some $\left.G \in C^{\infty}\left(\mathbf{R}^{d-1}\right)\right\}$. In the above and in what follows, we use the notation $a^{\beta}(x)$ instead of $a(x)$ to emphasize the dependency on $\boldsymbol{\beta}$. Since bilinear forms associated with $a^{\beta}(x)$ are calculated as (see Lemma A.1 in Appendix A)

$$
\left(a^{\boldsymbol{\beta}}(x) f, g\right)=\langle\bar{x}, \boldsymbol{\beta}(\hat{f}-\langle\bar{x}, \hat{f}\rangle \mathbf{1})(\hat{g}-\langle\bar{x}, \hat{g}\rangle \mathbf{1})\rangle, \quad f, g \in \mathbf{R}^{d-1},
$$

it follows from (3.3) that the forms $\mathcal{E}_{\boldsymbol{q}, C}^{\boldsymbol{\beta}}$ and $\mathcal{E}_{\boldsymbol{q} \boldsymbol{\beta}^{-1}, 0}^{\mathbf{1}}$ are equivalent in the sense that

$$
c_{1} \mathcal{E}_{\boldsymbol{q} \boldsymbol{\beta}^{-1}, 0}^{\mathbf{1}}(F, F) \leqslant \mathcal{E}_{\boldsymbol{q}, C}^{\boldsymbol{\beta}}(F, F) \leqslant c_{2} \mathcal{E}_{\boldsymbol{q} \boldsymbol{\beta}^{-1}, 0}^{\mathbf{1}}(F, F), \quad F \in C^{\infty}\left(K_{d}\right)
$$


for some positive finite constants $c_{1}=c_{1}(\boldsymbol{\beta}, \boldsymbol{q}, C)$ and $c_{2}=c_{2}(\boldsymbol{\beta}, \boldsymbol{q}, C)$. Because the form $\left(\mathcal{E}_{\boldsymbol{q} \boldsymbol{\beta}^{-1}, 0}^{\mathbf{1}}, C^{\infty}\left(K_{d}\right)\right)$ is closable in $L^{2}\left(D_{\boldsymbol{q} \boldsymbol{\beta}^{-1}}\right)$ (see [25, p. 670]), $\left(\mathcal{E}_{\boldsymbol{q}, C}^{\boldsymbol{\beta}}, C^{\infty}\left(K_{d}\right)\right)$ is closable in $L^{2}\left(P_{\boldsymbol{\beta}, \boldsymbol{q}, C}\right)$ and the closures of these two forms have a common domain. The logarithmic Sobolev inequalities obtained by Stannat is as follows.

Theorem 3.1 (Stannat, [25], Theorem 2.8). Let $\boldsymbol{q} \in \mathbf{R}_{+}^{d}$. For any $F \in C^{\infty}\left(K_{d}\right)$,

$$
\int_{K_{d}^{+}} F(x)^{2} \log \left(F(x)^{2}\right) D_{\boldsymbol{q}}(d x) \leqslant \frac{320}{\min \left\{q_{1}, \ldots, q_{d}\right\}} \mathcal{E}_{\boldsymbol{q}, 0}^{\mathbf{1}}(F, F)+\|F\|_{L^{2}\left(D_{\boldsymbol{q}}\right)}{ }^{2} \log \left(\|F\|_{L^{2}\left(D_{\boldsymbol{q}}\right)}^{2}\right) .
$$

By virtue of a uniform bound (3.3), we can generalize this result.

Proposition 3.2. Let $\boldsymbol{\beta}, \boldsymbol{q} \in \mathbf{R}_{+}^{d}$ and $C \in \mathbf{R}$. Then there exists a finite constant $c=c(\boldsymbol{\beta}, \boldsymbol{q}, C)$ such that for any $F \in C^{\infty}\left(K_{d}\right)$

$$
\int_{K_{d}^{+}} F(x)^{2} \log \left(F(x)^{2}\right) P_{\boldsymbol{\beta}, \boldsymbol{q}, C}(d x) \leqslant c \mathcal{E}_{\boldsymbol{q}, C}^{\boldsymbol{\beta}}(F, F)+\|F\|_{L^{2}\left(P_{\boldsymbol{\beta}, \boldsymbol{q}, C}\right)}{ }^{2} \log \left(\|F\|_{L^{2}\left(P_{\boldsymbol{\beta}, \boldsymbol{q}, C}\right)^{2}}\right) .
$$

Proof. By the argument in the proof of Lemma (3.13) in [12] (or by Property 4.6 in [8]), one can see from (3.3) existence of a finite constant $c_{3}=c_{3}(\boldsymbol{\beta}, \boldsymbol{q}, C)$ such that

$$
\int_{K_{d}^{+}} F(x)^{2} \log \left(\frac{F(x)^{2}}{\|F\|_{L^{2}\left(P_{\boldsymbol{\beta}, \boldsymbol{q}, C}\right)}^{2}}\right) P_{\boldsymbol{\beta}, \boldsymbol{q}, C}(d x) \leqslant c_{3} \int_{K_{d}^{+}} F(x)^{2} \log \left(\frac{F(x)^{2}}{\|F\|_{L^{2}\left(D_{\boldsymbol{q} \boldsymbol{\beta}^{-1}}\right)^{2}}}\right) D_{\boldsymbol{q} \boldsymbol{\beta}^{-1}}(d x)
$$

whenever $F \in L^{2}\left(D_{\boldsymbol{q} \boldsymbol{\beta}^{-1}}\right)=L^{2}\left(P_{\boldsymbol{\beta}, \boldsymbol{q}, C}\right)$. The inequality (3.16) follows from (3.14) and (3.15).

\section{Acknowledgements}

The author would like to thank the referee for valuable comments, which helped him improve considerably the presentation of an earlier version of the manuscript.

\section{Appendix A. Miscellanious calculations for the diffusion matrix}

This appendix is devoted to show basic facts of the diffusion matrix $a(x)$ of $L$, some of which are already used in the preceding sections. Let $\boldsymbol{\beta}=\left(\beta_{1}, \ldots, \beta_{d}\right)^{\prime} \in \mathbf{R}_{+}^{d}$. For each $x \in K_{d}$, consider a $(d-1) \times(d-1)$-matrix $a(x)=\left(a_{i j}(x)\right)_{1 \leqslant i, j \leqslant d-1}$ and a $d \times d$-matrix $\bar{a}(x)=\left(a_{i j}(x)\right)_{1 \leqslant i, j \leqslant d}$, where

$$
a_{i j}(x)=\delta_{i j} \beta_{i} x_{i}+x_{i} x_{j}\left(\langle\bar{x}, \boldsymbol{\beta}\rangle-\beta_{i}-\beta_{j}\right) .
$$

As before, set $\hat{f}=\left(f_{1}, \ldots, f_{d-1}, 0\right)^{\prime} \in \mathbf{R}^{d}$ for $f=\left(f_{1}, \ldots, f_{d-1}\right)^{\prime} \in \mathbf{R}^{d-1}$, and let $I=\{1, \ldots, d\}$. Bilinear forms associated with these matrices are given in the following lemma.

Lemma A.1. For each $x \in K_{d}$,

$$
(a(x) f, g)=\langle\bar{x}, \boldsymbol{\beta}(\hat{f}-\langle\bar{x}, \hat{f}\rangle \mathbf{1})(\hat{g}-\langle\bar{x}, \hat{g}\rangle \mathbf{1})\rangle, \quad f, g \in \mathbf{R}^{d-1}
$$

and 


$$
\begin{aligned}
\langle\bar{a}(x) \boldsymbol{\xi}, \boldsymbol{\eta}\rangle & =\langle\bar{x}, \boldsymbol{\beta} \boldsymbol{\xi} \boldsymbol{\eta}\rangle-\langle\bar{x}, \boldsymbol{\beta} \boldsymbol{\xi}\rangle\langle\bar{x}, \boldsymbol{\eta}\rangle-\langle\bar{x}, \boldsymbol{\xi}\rangle\langle\bar{x}, \boldsymbol{\beta} \boldsymbol{\eta}\rangle+\langle\bar{x}, \boldsymbol{\beta}\rangle\langle\bar{x}, \boldsymbol{\xi}\rangle\langle\bar{x}, \boldsymbol{\eta}\rangle \\
& =\langle\bar{x}, \boldsymbol{\beta}(\boldsymbol{\xi}-\langle\bar{x}, \boldsymbol{\xi}\rangle \mathbf{1})(\boldsymbol{\eta}-\langle\bar{x}, \boldsymbol{\eta}\rangle \mathbf{1})\rangle, \quad \boldsymbol{\xi}, \boldsymbol{\eta} \in \mathbf{R}^{d} .
\end{aligned}
$$

Proof. In view of $(a(x) f, g)=\langle\bar{a}(x) \hat{f}, \hat{g}\rangle$, it is sufficient to show (A.3) only. This is easily done by considering the case where $\boldsymbol{\xi}=\boldsymbol{\varepsilon}^{(i)}, \boldsymbol{\eta}=\boldsymbol{\varepsilon}^{(j)}(i, j \in I)$. (Here $\boldsymbol{\varepsilon}^{(i)}=\left(\delta_{i k}\right)_{k=1}^{d}, i=1, \ldots, d$.) We omit the details.

It is not difficult to see from Lemma A.1 that $a(x)$ is degenerate if $x_{i}=0$ for some $i \in I$ and that $\bar{a}(x)$ is always degenerate. The following proposition shows these facts explicitly.

\section{Proposition A.1.}

$$
\operatorname{det} a(x)=\left(\beta_{1} x_{1}\right) \cdots\left(\beta_{d} x_{d}\right)\left\langle\bar{x}, \boldsymbol{\beta}^{-1}\right\rangle .
$$

Proof. Since $\operatorname{det} a(x)$ is continuous in $x$, we can assume that $x \in K_{d}^{+}$, i.e., $x_{i}>0$ for all $i \in I$. For notational simplicity, set $n=d-1$ and $b=\langle\bar{x}, \boldsymbol{\beta}\rangle$. First observe that

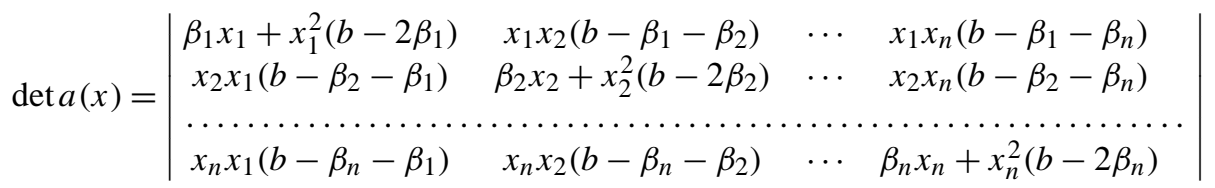

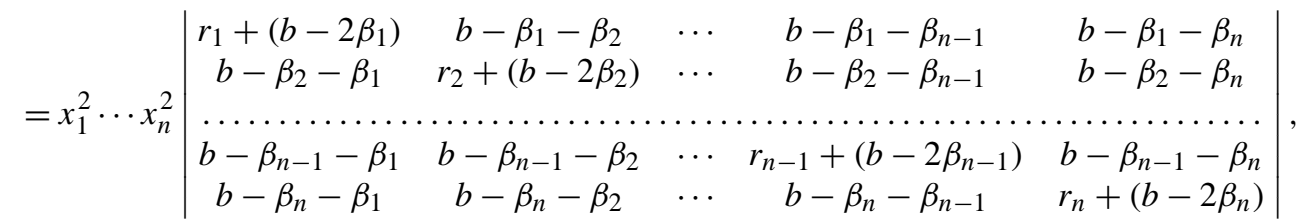

where $r_{i}=\beta_{i} / x_{i}$. Let $f^{(i)}$ be the $i$ th row vector in the above. Subtract $f^{(i-1)}$ from $f^{(i)}(i=n, n-1, \ldots, 2)$ to get

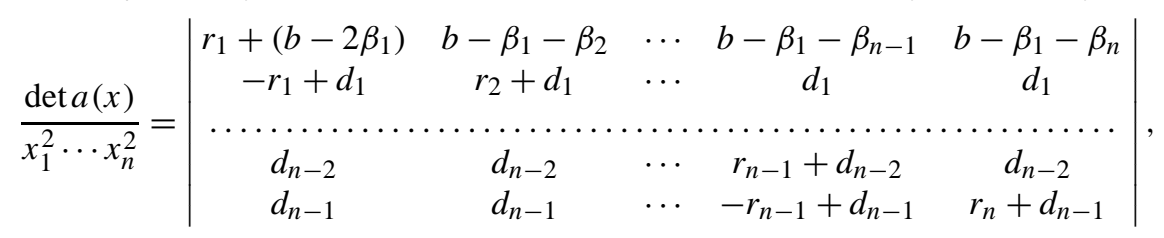

where $d_{i}=\beta_{i}-\beta_{i+1}$. Denoting by $g^{(j)}$ the $j$ th column vector in the above, subtract $g^{(j-1)}$ from $g^{(j)}(j=$ $n, n-1, \ldots, 2)$ to get

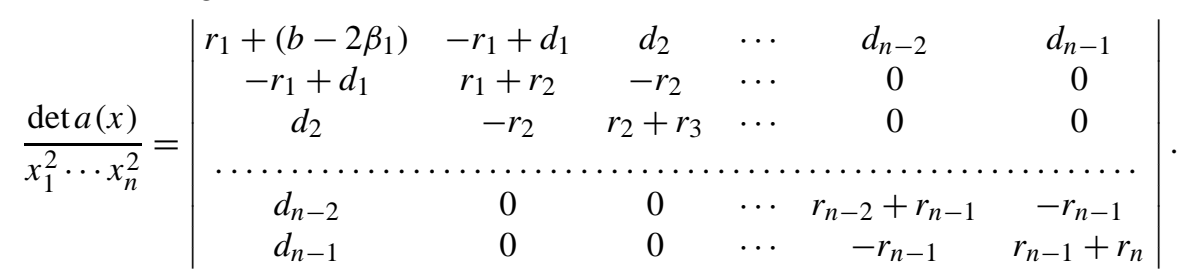

Let $h^{(i)}$ be the $i$ th row vector in the above. Add $h^{(2)}+\cdots+h^{(i-1)}$ to $h^{(i)}(i=3, \ldots, n)$. Then

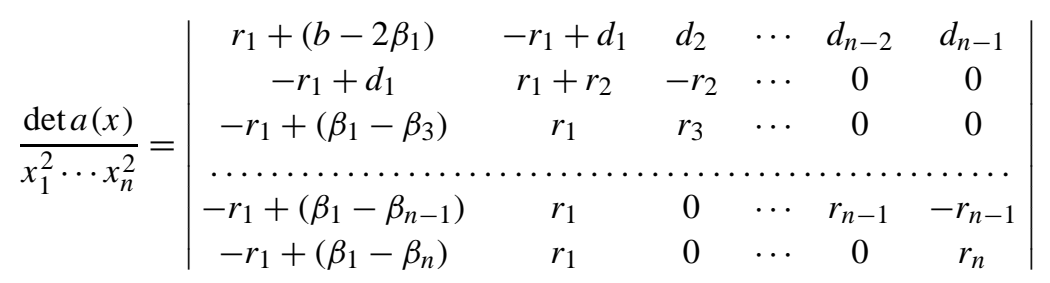




$$
\begin{aligned}
& =\left|\begin{array}{cccccc}
b-\beta_{1}-\beta_{2} & -r_{1}+d_{1} & d_{2} & \cdots & d_{n-2} & d_{n-1} \\
r_{2}+d_{1} & r_{1}+r_{2} & -r_{2} & \cdots & 0 & 0 \\
\beta_{1}-\beta_{3} & r_{1} & r_{3} & \cdots & 0 & 0 \\
\ldots \ldots \ldots \ldots \ldots \ldots \ldots \ldots \ldots \ldots \ldots \ldots & \ldots \ldots \ldots \ldots \ldots \\
\beta_{1}-\beta_{n-1} & r_{1} & 0 & \cdots & r_{n-1} & -r_{n-1} \\
\beta_{1}-\beta_{n} & r_{1} & 0 & \cdots & 0 & r_{n}
\end{array}\right|
\end{aligned}
$$

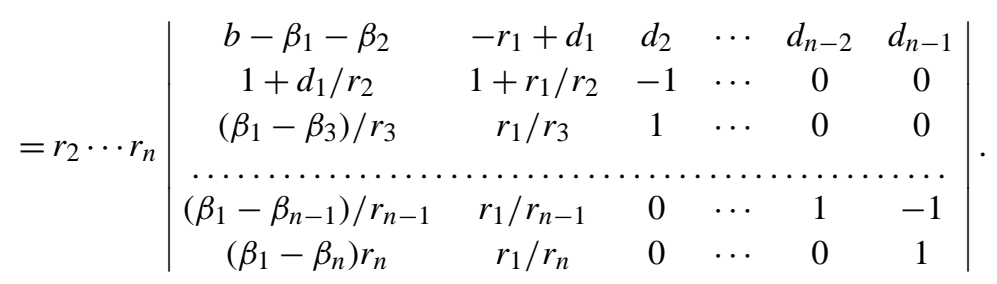

Putting $s_{k}=\left(\beta_{1}-\beta_{k}\right) / r_{k}$ and $t_{k}=r_{1} / r_{k}$, we have

$$
\begin{aligned}
& \frac{\operatorname{det} a(x)}{x_{1}^{2}\left(\beta_{2} x_{2}\right) \cdots\left(\beta_{n} x_{n}\right)}=\left|\begin{array}{cccccc}
b-\beta_{1}-\beta_{2} & -r_{1}+d_{1} & d_{2} & \cdots & d_{n-2} & d_{n-1} \\
1+s_{2} & 1+t_{2} & -1 & \cdots & 0 & 0 \\
s_{3} & t_{3} & 1 & \ldots & 0 & 0 \\
\ldots \ldots \ldots \ldots \ldots \ldots \ldots \ldots \ldots \ldots & \ldots \ldots \ldots \ldots \ldots \ldots \\
s_{n-1} & t_{n-1} & 0 & \cdots & 1 & -1 \\
s_{n} & t_{n} & 0 & \cdots & 0 & 1
\end{array}\right| \\
& =\left|\begin{array}{cccccc}
b-\beta_{1}-\beta_{2} & -r_{1}+d_{1} & d_{2} & \cdots & d_{n-2} & d_{n-1} \\
1+\sum_{k=2}^{n} s_{k} & 1+\sum_{k=2}^{n} t_{k} & 0 & \cdots & 0 & 0 \\
\sum_{k=3}^{n} s_{k} & \sum_{k=3}^{n} t_{k} & 1 & \ldots & 0 & 0 \\
\ldots \ldots \ldots \ldots \ldots \ldots \ldots \ldots \ldots & \ldots \ldots \ldots \ldots \ldots \ldots \\
s_{n-1}+s_{n} & t_{n-1}+t_{n} & 0 & \cdots & 1 & 0 \\
s_{n} & t_{n} & 0 & \cdots & 0 & 1
\end{array}\right|,
\end{aligned}
$$

which can be reduced to a $2 \times 2$ determinant

$$
\left|\begin{array}{cc}
\left(b-\beta_{1}-\beta_{2}\right)-\sum_{l=3}^{n}\left(\sum_{k=l}^{n} s_{k}\right) d_{l-1} & \left(-r_{1}+d_{1}\right)-\sum_{l=3}^{n}\left(\sum_{k=l}^{n} t_{k}\right) d_{l-1} \\
1+\sum_{k=2}^{n} s_{k} & 1+\sum_{k=2}^{n} t_{k}
\end{array}\right| .
$$

Further calculations are messy and left to readers.

Proposition A.2. Let $x \in K_{d}^{+}$. Then for $f, g \in \mathbf{R}^{d-1}$

$$
\begin{aligned}
\left(a(x)^{-1} f, g\right)= & \frac{\left\langle\hat{x}, \boldsymbol{\beta}^{-1}\right\rangle}{\left\langle\bar{x}, \boldsymbol{\beta}^{-1}\right\rangle}\left\langle\hat{x}, \boldsymbol{\beta}^{-1}\left\{\left(\frac{\hat{f}}{x}\right)-\frac{\left\langle\boldsymbol{\beta}^{-1}, \hat{f}\right\rangle}{\left\langle\hat{x}, \boldsymbol{\beta}^{-1}\right\rangle} \mathbf{1}\right\}\left\{\left(\frac{\hat{g}}{x}\right)-\frac{\left\langle\boldsymbol{\beta}^{-1}, \hat{g}\right\rangle}{\left\langle\hat{x}, \boldsymbol{\beta}^{-1}\right\rangle} \mathbf{1}\right\}\right\rangle \\
& +\frac{x_{d} \beta_{d}^{-1}}{\left\langle\bar{x}, \boldsymbol{\beta}^{-1}\right\rangle}\left\langle\hat{x}, \boldsymbol{\beta}^{-1}\left\{\left(\frac{\hat{f}}{x}\right)+\frac{\langle\mathbf{1}, \hat{f}\rangle}{x_{d}} \mathbf{1}\right\}\left\{\left(\frac{\hat{g}}{x}\right)+\frac{\langle\mathbf{1}, \hat{g}\rangle}{x_{d}} \mathbf{1}\right\}\right),
\end{aligned}
$$


where

$$
\left(\frac{\hat{f}}{x}\right)=\left(\frac{f_{1}}{x_{1}}, \ldots, \frac{f_{d-1}}{x_{d-1}}, 0\right)^{\prime} .
$$

In particular, $a(x)^{-1}=:\left(a^{i j}(x)\right)_{1 \leqslant i, j \leqslant d-1}$ is given by

$$
a^{i j}(x)=\frac{\delta_{i j}}{x_{i} \beta_{i}}+\frac{1}{x_{d} \beta_{d}}-\left(\frac{1}{\beta_{i}}-\frac{1}{\beta_{d}}\right)\left(\frac{1}{\beta_{j}}-\frac{1}{\beta_{d}}\right) \frac{1}{\left\langle\bar{x}, \boldsymbol{\beta}^{-1}\right\rangle} .
$$

\section{Corollary A.1. (i)}

$$
(a(x) f, f) \geqslant(2 d)^{-1} \min _{1 \leqslant i \leqslant d}\left(x_{i} \beta_{i}\right)(f, f), \quad f \in \mathbf{R}^{d-1} .
$$

(ii) Define $U: K_{d}^{+} \longrightarrow \mathbf{R}$ by

$$
U(x)=\sum_{i=1}^{d} x_{i} \beta_{i}^{-1} \log \frac{x_{i} \beta_{i}^{-1}}{\left\langle\bar{x}, \boldsymbol{\beta}^{-1}\right\rangle} .
$$

Then

$$
\frac{\partial^{2} U}{\partial x_{i} \partial x_{j}}=a^{i j}(x), \quad x \in K_{d}^{+}, i, j \in\{1, \ldots, d-1\} .
$$

Proof. (i) To avoid triviality, we assume $x \in K_{d}^{+}$and put $M:=\max \left\{x_{i}{ }^{-1} \beta_{i}{ }^{-1}: i \in I\right\}$. Because of

$$
\frac{1}{2}\left(a(x)^{-1} g, g\right)=\sup _{f \in \mathbf{R}^{d-1}}\left\{(f, g)-\frac{1}{2}(a(x) f, f)\right\},
$$

it suffices to show that

$$
\left(a(x)^{-1} g, g\right) \leqslant 2 d M(g, g), \quad g \in \mathbf{R}^{d-1} .
$$

Expanding the right-hand side of (A.5), we see without difficulty

$$
\begin{aligned}
& \left(a(x)^{-1} g, g\right)\left\langle\bar{x}, \boldsymbol{\beta}^{-1}\right\rangle \\
& \quad \leqslant\left\langle\hat{x}, \boldsymbol{\beta}^{-1}\right\rangle\left\langle\bar{x}^{-1}, \boldsymbol{\beta}^{-1} \hat{g}^{2}\right\rangle+2 x_{d} \beta_{d}{ }^{-1}\left\{\left\langle\bar{x}^{-1}, \boldsymbol{\beta}^{-1} \hat{g}^{2}\right\rangle+\frac{\left\langle\hat{x}, \boldsymbol{\beta}^{-1}\right\rangle\langle\mathbf{1}, \hat{g}\rangle^{2}}{x_{d}{ }^{2}}\right\} \\
& \quad \leqslant 2\left\langle\bar{x}, \boldsymbol{\beta}^{-1}\right\rangle\left\langle\bar{x}^{-1}, \boldsymbol{\beta}^{-1} \hat{g}^{2}\right\rangle+2 \frac{\left\langle\bar{x}, \boldsymbol{\beta}^{-1}\right\rangle}{x_{d} \beta_{d}}\langle\mathbf{1}, \hat{g}\rangle^{2},
\end{aligned}
$$

and hence

$$
\begin{aligned}
\left(a(x)^{-1} g, g\right) / 2 & \leqslant\left\langle\bar{x}^{-1}, \boldsymbol{\beta}^{-1} \hat{g}^{2}\right\rangle+x_{d}^{-1} \beta_{d}{ }^{-1}\langle\mathbf{1}, \hat{g}\rangle^{2} \\
& \leqslant M(g, g)+M(d-1)(g, g)=d M(g, g) .
\end{aligned}
$$

(ii) (A.8) is immediate from

$$
\frac{\partial U}{\partial x_{i}}=\frac{\log \left(x_{i} \beta_{i}^{-1}\right)}{\beta_{i}}-\frac{\log \left(x_{d} \beta_{d}^{-1}\right)}{\beta_{d}}-\left(\frac{1}{\beta_{i}}-\frac{1}{\beta_{d}}\right) \log \left\langle\bar{x}, \boldsymbol{\beta}^{-1}\right\rangle .
$$

Proof of Proposition A.2. Although one can check the validity of (A.5) and (A.6) directly, we shall give a sketch of their derivation. Let $g \in \mathbf{R}^{d-1}$ and $x \in K_{d}$ be given. Note that the supremum in (A.9) is attained at $f=a(x)^{-1} g$. By Lemma A.1

$$
(a(x) f, f)=(x, f)^{2}\langle\bar{x}, \boldsymbol{\beta}\rangle-2(x, f)\langle\bar{x}, \boldsymbol{\beta} \hat{f}\rangle+\left\langle\bar{x}, \boldsymbol{\beta} \hat{f}^{2}\right\rangle .
$$


Considering

$$
G(f):=(f, g)-\frac{1}{2}(a(x) f, f),
$$

observe that the maximizer $f$ of $G$ must satisfy

$$
0=g_{i}-(x, f)\langle\bar{x}, \boldsymbol{\beta}\rangle x_{i}+\langle\bar{x}, \boldsymbol{\beta} \hat{f}\rangle x_{i}+(x, f) \beta_{i} x_{i}-\beta_{i} f_{i} x_{i}=: \partial_{i} G(f), \quad i=1, \ldots, d-1 .
$$

Computing $\sum_{i} \partial_{i} G(f)$ and $\sum_{i} \partial_{i} G(f) / \beta_{i}$, one can derive the following equalities which together determine $A:=(x, f)$ and $B:=\langle\bar{x}, \boldsymbol{\beta} \hat{f}\rangle$ in terms of $x, \boldsymbol{\beta}$ and $g$.

$$
\begin{aligned}
& \left\{\left(\beta_{d}-\langle\bar{x}, \boldsymbol{\beta}\rangle\right) x_{d}\right\} A+x_{d} B=\langle\mathbf{1}, \hat{g}\rangle, \\
& \left\{\left\langle\hat{x}, \boldsymbol{\beta}^{-1}\right\rangle\langle\bar{x}, \boldsymbol{\beta}\rangle+x_{d}\right\} A-\left\langle\hat{x}, \boldsymbol{\beta}^{-1}\right\rangle B=\left\langle\boldsymbol{\beta}^{-1}, \hat{g}\right\rangle .
\end{aligned}
$$

On the other hand, (A.10) implies that $f=a(x)^{-1} g$ is given by

$$
f_{i}=\frac{g_{i}}{x_{i} \beta_{i}}-\frac{\langle\bar{x}, \boldsymbol{\beta}\rangle}{\beta_{i}} A+\frac{B}{\beta_{i}}+A, \quad i=1, \ldots, d-1 .
$$

Using (A.11a), (A.11b) and (A.12), one can compute the quadratic form $\left(a(x)^{-1} g, g\right)=(f, g)$ to obtain (A.5) with $g$ in place of $f$. Remaining calculations are straightforward and omitted.

\section{Appendix B. Transformation group associated with the diffusion matrix}

In this appendix, we construct a transformation group $\left\{S_{f}: f \in \mathbf{R}^{d-1}\right\}$ on $K_{d}$ such that

$$
\frac{d}{d u} S_{u f} x=a\left(S_{u f} x\right) f, \quad f=\left(f_{1}, \ldots, f_{d-1}\right)^{\prime} \in \mathbf{R}^{d-1}, x \in K_{d}
$$

and show the properties (S.1)-(S.8) used in Section 2. Firstly, extend $a_{i j}(x)$ as a bounded smooth function on $\mathbf{R}^{d-1}$ such that

$$
a_{i j}(x)= \begin{cases}\delta_{i j} \beta_{i} x_{i}+x_{i} x_{j}\left(\langle\bar{x}, \boldsymbol{\beta}\rangle-\beta_{i}-\beta_{j}\right), & \operatorname{dist}\left(x, K_{d}\right) \leqslant 1, \\ 0, & \operatorname{dist}\left(x, K_{d}\right) \geqslant 2,\end{cases}
$$

where $\bar{x}=\left(x_{1}, \ldots, x_{d-1}, x_{d}\right)^{\prime}$ with $x_{d}=1-x_{1}-\cdots-x_{d-1}$. Then $a(x)=\left(a_{i j}(x)\right)_{1 \leqslant i, j \leqslant d-1}$ satisfies Lipschitz condition

$$
\|a(x) f-a(y) f\|_{1} \leqslant C\|f\|_{1}\|x-y\|_{1}, \quad x, y, f \in \mathbf{R}^{d-1}
$$

for some constant $C$, where $\|f\|_{1}=\left|f_{1}\right|+\cdots+\left|f_{d-1}\right|$ etc. Therefore, given $f \in \mathbf{R}^{d-1}$ and $x \in \mathbf{R}^{d-1}$, one can get a unique global solution $X(t) \in \mathbf{R}^{d-1}(t \in \mathbf{R})$ of an ODE

$$
\frac{d}{d t} X(t)=a(X(t)) f, \quad X(0)=x
$$

by a standard successive approximation $X^{(n)}(t) \rightarrow X(t)(n \rightarrow \infty)$, where

$$
X^{(n)}(t)=x+\int_{0}^{t} a\left(X^{(n-1)}(s)\right) f d s, \quad X^{(0)}(t)=x .
$$

Moreover, setting $C^{\prime}=(d-1) \sup \left\{\left|a_{i j}(x)\right|: x \in \mathbf{R}^{d-1}, 1 \leqslant i, j \leqslant d-1\right\}$, we have

$$
\|X(t)\|_{1} \leqslant\|x\|_{1}+C^{\prime}\|f\|_{1}|t|, \quad t \in \mathbf{R} .
$$


Let $T_{t}^{f}$ denote the flow associated with (A.15); $T_{t}^{f} x=X(t)$. For each $x \in K_{d}$, define $S_{f} x=T_{1}^{f} x$. The next lemma shows not only that $S_{f}: K_{d} \rightarrow K_{d}$ but also (S.1) and (S.3).

Lemma A.2. Let $f \in \mathbf{R}^{d-1}$ and $x \in K_{d}$. Suppose that $X(t)=\left(X_{1}(t), \ldots, X_{d-1}(t)\right)^{\prime}$ solves (A.15). Put $X_{d}(t)=$ $1-\left(X_{1}(t)+\cdots+X_{d-1}(t)\right)$. Then $X(t) \in K_{d}(t \in \mathbf{R})$, and for any $i \in I$,

(i) $X_{i}(t)=0, t \in \mathbf{R}$ whenever $x_{i}=0$, and

(ii) $X_{i}(t)>0, t \in \mathbf{R}$ whenever $x_{i}>0$.

Proof. Because of an obvious relation $T_{-t}^{f} x=T_{t}^{-f} x$, we only have to consider $t>0$ without any loss of generality. Let $x \in K_{d}$. In order to show that $X(t) \in K_{d}$ for all $t>0$, assume that $t_{0}:=\inf \left\{t>0: X(t) \notin K_{d}\right\}<\infty$. By continuity, we can find also $t_{1}>t_{0}$ such that $\operatorname{dist}\left(X(t), K_{d}\right) \leqslant 1$ for all $0 \leqslant t \leqslant t_{1}$. On the other hand, it follows from the definition of $t_{0}$ that there exist $i_{0} \in I$ and $t^{\prime} \in\left(t_{0}, t_{1}\right)$ such that $X_{i_{0}}\left(t_{0}\right)=0$ and $X_{i_{0}}\left(t^{\prime}\right)<0$. In the case where $i_{0} \in\{1, \ldots, d-1\}$, for any $t \in\left[t_{0}, t_{1}\right]$

$$
\begin{aligned}
X_{i_{0}}(t) & =X_{i_{0}}\left(t_{0}\right)+\int_{t_{0}}^{t} \sum_{j=1}^{d-1} a_{i_{0} j}(X(s)) f_{j} d s \\
& =\int_{t_{0}}^{t} X_{i_{0}}(s) \sum_{j=1}^{d-1}\left\{X_{j}(s)\left(\langle\overline{X(s)}, \boldsymbol{\beta}\rangle-\beta_{i_{0}}-\beta_{j}\right) f_{j}+\beta_{i_{0}} f_{i_{0}}\right\} d s .
\end{aligned}
$$

With the help of (A.16), we get

$$
\left|X_{i_{0}}(t)\right| \leqslant C_{1} \int_{t_{0}}^{t}\left|X_{i_{0}}(s)\right|(1+s)^{2} d s, \quad t \in\left[t_{0}, t_{1}\right]
$$

for some constant $C_{1}$. So by Gronwall's inequality (see e.g. [3], Appendix 5) $X_{i_{0}}(t)=0, t \in\left[t_{0}, t_{1}\right]$, which contradicts to that $X_{i_{0}}\left(t^{\prime}\right)<0$. In the case of $i_{0}=d$, for any $t \in\left[t_{0}, t_{1}\right]$

$$
\begin{aligned}
X_{d}(t)=1-\sum_{i=1}^{d-1} X_{i}(t) & =X_{d}\left(t_{0}\right)-\int_{t_{0}}^{t} \sum_{i, j=1}^{d-1} a_{i j}(X(s)) f_{j} d s \\
& =-\int_{t_{0}}^{t} X_{d}(s) \sum_{j=1}^{d-1} X_{j}(s)\left(\langle\overline{X(s)}, \boldsymbol{\beta}\rangle-\beta_{d}-\beta_{j}\right) f_{j} d s .
\end{aligned}
$$

Again by (A.16) and Gronwall's inequality, $X_{d}(t)=0, t \in\left[t_{0}, t_{1}\right]$, which contradicts to that $X_{d}\left(t^{\prime}\right)<0$. Consequently, $t_{0}$ cannot be finite, or equivalently, $X(t) \in K_{d}$ for all $t>0$.

Clearly, the assertion (i) can be proved in the same way as above, and it remains to prove (ii). First consider the case where $1 \leqslant i \leqslant d-1$ and $x_{i}>0$. Let $\tau=\inf \left\{t>0: X_{i}(t)=0\right\}>0$. Then for $0<t<\tau$

$$
\begin{aligned}
\left|\log X_{i}(t)\right| & \leqslant\left|\log x_{i}\right|+\int_{0}^{t}\left|X_{i}(s)^{-1} \sum_{j=1}^{d-1} a_{i j}(X(s)) f_{j}\right| d s \\
& =\left|\log x_{i}\right|+\int_{0}^{t}\left|\sum_{j=1}^{d-1} X_{j}(s)\left(\langle\overline{X(s)}, \boldsymbol{\beta}\rangle-\beta_{i}-\beta_{j}\right) f_{j}+\beta_{i} f_{i}\right| d s .
\end{aligned}
$$


Note that the most right-hand side cannot diverge for any finite value of $t$ because of (A.16). This proves that $\tau=\infty$. Thus $X_{i}(t)>0$ for all $t>0$. One can handle the remaining case where $x_{d}>0$ by just the same way, observing that

$$
\begin{aligned}
\left|\log X_{d}(t)\right| & \leqslant\left|\log x_{d}\right|+\int_{0}^{t}\left|-X_{d}(s)^{-1} \sum_{i, j=1}^{d-1} a_{i j}(X(s)) f_{j}\right| d s \\
& =\left|\log x_{d}\right|+\int_{0}^{t}\left|\sum_{j=1}^{d-1} X_{j}(s)\left(\langle\overline{X(s)}, \boldsymbol{\beta}\rangle-\beta_{d}-\beta_{j}\right) f_{j}\right| d s
\end{aligned}
$$

as long as $X_{d}(s)>0$ for all $s \in[0, t]$.

The property (S.7) follows from the next lemma.

Lemma A.3. Let $C$ and $C^{\prime}$ be as in (A.14) and (A.16), respectively. For any $x, y \in \mathbf{R}^{d-1}, f, g \in \mathbf{R}^{d-1}$ and $t \in \mathbf{R}$

$$
\left\|T_{t}^{f} x-T_{t}^{g} y\right\|_{1} \leqslant\left(\|x-y\|_{1}+C^{\prime}\|f-g\|_{1}|t|\right) e^{C|t| \min \left\{\|f\|_{1},\|g\|_{1}\right\}} .
$$

Proof. As before, we may assume that $t>0$. It is seen from (A.14) and (A.16) that

$$
\begin{aligned}
\left\|T_{t}^{f} x-T_{t}^{g} x\right\|_{1} & \leqslant \int_{0}^{t}\left\|a\left(T_{u}^{f} x\right) f-a\left(T_{u}^{g} x\right) g\right\|_{1} d u \\
& \leqslant C^{\prime}\|f-g\|_{1} t+C\left\|_{g}\right\|_{1} \int_{0}^{t}\left\|T_{u}^{f} x-T_{u}^{g} x\right\|_{1} d u .
\end{aligned}
$$

By Gronwall's inequality

$$
\left\|T_{t}^{f} x-T_{t}^{g} x\right\|_{1} \leqslant C^{\prime}\|f-g\|_{1}\left(t+\int_{0}^{t} u C\|g\|_{1} e^{C\|g\|_{1}(t-u)} d u\right) .
$$

Also,

$$
\begin{aligned}
\left\|T_{t}^{g} x-T_{t}^{g} y\right\|_{1} & \leqslant\|x-y\|_{1}+\int_{0}^{t}\left\|a\left(T_{u}^{g} x\right) g-a\left(T_{u}^{g} y\right) g\right\|_{1} d u \\
& \leqslant\|x-y\|_{1}+C\|g\|_{1} \int_{0}^{t}\left\|T_{u}^{g} x-T_{u}^{g} y\right\|_{1} d u .
\end{aligned}
$$

Again by Gronwall's inequality

$$
\left\|T_{t}^{g} x-T_{t}^{g} y\right\|_{1} \leqslant\|x-y\|_{1}\left(1+\int_{0}^{t} C\|g\|_{1} e^{C\|g\|_{1}(t-u)} d u\right) .
$$

Combining (A.18) with (A.19), one obtains 


$$
\begin{aligned}
\left\|T_{t}^{f} x-T_{t}^{g} y\right\|_{1} & \leqslant\left(\|x-y\|_{1}+C^{\prime}\|f-g\|_{1} t\right)\left(1+\int_{0}^{t} C\|g\|_{1} e^{C\|g\|_{1}(t-u)} d u\right) \\
& =\left(\|x-y\|_{1}+C^{\prime}\|f-g\|_{1} t\right) e^{C\|g\|_{1} t} .
\end{aligned}
$$

Exchanging the roll of $f$ and $g$ yields

$$
\left\|T_{t}^{f} x-T_{t}^{g} y\right\|_{1} \leqslant\left(\|x-y\|_{1}+C^{\prime}\|f-g\|_{1} t\right) e^{C\|f\|_{1} t} .
$$

The above two inequalities together prove (A.17).

Now we introduce a map which will play a key role in the argument below. Letting $U: K_{d}^{+} \rightarrow \mathbf{R}$ be the function given in Corollary A.1(ii), define $\Phi: K_{d}^{+} \rightarrow \mathbf{R}^{d-1}$ by $\Phi=\nabla U$. (S.2) and (S.6) are implied by the next proposition.

Proposition A.3. Let $f, g \in \mathbf{R}^{d-1}$ and $t \in \mathbf{R}$.

(i) $T_{u}^{t f} x=T_{u t}^{f} x\left(x \in \mathbf{R}^{d-1}, u \in \mathbf{R}\right)$. In particular, $S_{t f} x=T_{t}^{f} x\left(x \in K_{d}\right)$.

(ii) $T_{t}^{f} x$ is continuously differentiable in $x \in \mathbf{R}^{d-1}$ and its Jacobian matrix $D_{x}\left(T_{t}^{f} x\right)$ satisfies

$$
D_{x}\left(T_{t}^{f} x\right) a(x)=a\left(T_{t}^{f} x\right), \quad x \in K_{d} .
$$

(iii)

$$
T_{t}^{f}\left(T_{t}^{g} x\right)=T_{t}^{f+g} x, \quad x \in K_{d}
$$

Proof. The first assertion follows immediately from

$$
\frac{d}{d u} T_{u t}^{f} x=\operatorname{ta}\left(T_{u t}^{f} x\right) f=a\left(T_{u t}^{f} x\right)(t f) .
$$

Since $T_{t}^{f} x$ is given as the strong limit of successive approximations

$$
T_{t}^{(n)} x=x+\int_{0}^{t} a\left(T_{s}^{(n-1)} x\right) f d s, \quad T^{(0)}(t)=x,
$$

it is not difficult to show that $T_{t}^{f} x=\lim T_{t}^{(n)} x$ is continuously differentiable in $x \in \mathbf{R}^{d-1}$. To show (A.20), we may assume $x \in K_{d}^{+}$because of continuity. By (A.8) the Jacobian matrix $D \Phi(x)$ of $\Phi$ at $x \in K_{d}^{+}$is given by

$$
D \Phi(x)=a(x)^{-1}, \quad x \in K_{d}^{+} .
$$

We claim that $\Phi$ is an injection. This is because for any $x, y \in K_{d}^{+}$

$$
\begin{aligned}
(\Phi(x)-\Phi(y), x-y) & =\int_{0}^{1}(D \Phi(y+u(x-y))(x-y), x-y) d u \\
& =\int_{0}^{1}\left(a(y+u(x-y))^{-1}(x-y), x-y\right) d u
\end{aligned}
$$

which vanishes only when $x=y$. Furthermore, observe from (A.22) that for any $x \in K_{d}^{+}$

$$
\frac{d}{d t} \Phi\left(T_{t}^{f} x\right)=\left.D \Phi(y)\right|_{y=T_{t}^{f} x} a\left(T_{t}^{f} x\right) f=f
$$


or $\Phi\left(T_{t}^{f} x\right)=t f+\Phi(x)$. This proves not only that $\Phi$ is a surjection but also that

$$
T_{t}^{f} x=\Phi^{-1}(t f+\Phi(x)), \quad x \in K_{d}^{+} .
$$

Differentiating this equality with respect to $x \in K_{d}^{+}$, we get

$$
D_{x}\left(T_{t}^{f} x\right)=\left.D\left(\Phi^{-1}\right)(g)\right|_{g=t f+\Phi(x)} D \Phi(x)=\left(\left.D \Phi(y)\right|_{y=T_{t}^{f} x}\right)^{-1} a(x)^{-1}=a\left(T_{t}^{f} x\right) a(x)^{-1},
$$

proving (A.20). Lastly, (A.21) is easily seen from (A.23) for $x \in K_{d}^{+}$. It extends to all $x \in K_{d}$ by continuity. We complete the proof of Proposition A.3.

The property (S.5) is shown by Proposition A.3(iii). Indeed, putting $e^{(j)}=\left(\delta_{k j}\right)_{k=1}^{d-1}$ for each $j=1, \ldots, d-1$, we have by (A.21)

$$
\begin{aligned}
\frac{\partial\left(S_{f} x\right)_{i}}{\partial f_{j}} & =\left.\frac{d}{d u}\left(S_{f+u e^{(j)}} x\right)_{i}\right|_{u=0}=\left.\frac{d}{d u}\left(S_{u e^{(j)}}\left(S_{f} x\right)\right)_{i}\right|_{u=0} \\
& =\left.\frac{d}{d u}\left(T_{u}^{e^{(j)}}\left(S_{f} x\right)\right)_{i}\right|_{u=0}=\left(a\left(S_{f} x\right) e^{(j)}\right)_{i}=a_{i j}\left(S_{f} x\right) .
\end{aligned}
$$

The following result contains (S.4).

Proposition A.4. Let $x, y \in K_{d}^{+}$be arbitrary. Set $\check{\mathbf{0}}=(0, \ldots, 0)^{\prime} \in \mathbf{R}^{d-1}$.

(i) There exists a unique $f \in \mathbf{R}^{d-1}$ such that $y=S_{f} x$.

(ii) For any $f \in \mathbf{R}^{d-1} \backslash\{\check{\mathbf{0}}\}$, $\operatorname{dist}\left(S_{t f} x, \partial K_{d}\right) \rightarrow 0$ as $t \rightarrow \infty$, where $\partial K_{d}$ is the boundary of $K_{d}$ (in $\left.\mathbf{R}^{d-1}\right)$.

Proof. (i) First we show the uniqueness. Suppose that $S_{f} x=S_{g} x$ for some $f, g \in \mathbf{R}^{d-1}$. Then by the property (S.2) $h:=f-g$ satisfies $S_{h} x=x$. This together with (S.5) just seen above implies that

$$
0=\left(S_{h} x, h\right)-(x, h)=\int_{0}^{1}\left(a\left(S_{t h} x\right) h, h\right) d t .
$$

Since by Lemma A.2 $S_{t h} x \in K_{d}^{+}$for any $x \in K_{d}^{+}, a\left(S_{t h} x\right)$ is strictly positive definite and so we conclude that $h=\check{\mathbf{0}}$ or $f=g$. Next we shall find $f \in \mathbf{R}^{d-1}$ such that $S_{f} x=y$. Define $f=\Phi(y)-\Phi(x) \in \mathbf{R}^{d-1}$. It then follows from (A.23) that

$$
S_{f} x=T_{1}^{f} x=\Phi^{-1}(f+\Phi(x))=\Phi^{-1}(\Phi(y))=y
$$

as required.

(ii) Observe from (S.5), which has been proved, that

$$
\left(S_{t f} x, f\right)-(x, f)=\int_{0}^{t}\left(a\left(S_{u f} x\right) f, f\right) d u .
$$

Note that the left-hand side remains bounded as $t \rightarrow \infty$. As for the right-hand side, by (A.7)

$$
\left(a\left(S_{u f} x\right) f, f\right) \geqslant \frac{\min _{1 \leqslant i \leqslant d} \beta_{i}}{2 d} \min _{1 \leqslant i \leqslant d}\left(S_{u f} x\right)_{i}(f, f)^{2} \geqslant 0 .
$$

Therefore $\min _{1 \leqslant i \leqslant d}\left(S_{t f} x\right)_{i} \rightarrow 0$ as $t \rightarrow \infty$, provided that $f \neq \check{\mathbf{0}}$. This proves the second assertion. 
Our final task is to prove (S.8), which concerns a $\sigma$-finite measure

$$
m(d x)=d x_{1} \cdots d x_{d-1} / \operatorname{det} a(x)
$$

on $K_{d}^{+}$. In fact, it will be shown that $m(d x)$ is a Haar measure with respect to multiplications on $K_{d}^{+}$described as follows. As was seen in the proof of Proposition A.3, $\Phi: K_{d}^{+} \rightarrow \mathbf{R}^{d-1}$ is surjective. So it is possible to define

$$
x * y=\Phi^{-1}(\Phi(x)+\Phi(y)), \quad x, y \in K_{d}^{+} .
$$

Lemma A.4. (i) There exists a unique $e \in K_{d}^{+}$such that $x * e=x=e * x$ for all $x \in K_{d}^{+}$.

(ii) The inverse element $x^{*-1}$ of $x \in K_{d}^{+}$(i.e., a unique $y \in K_{d}^{+}$such that $x * y=e=y * x$ ) is given by

$$
x^{*-1}=S_{-\Phi(x)} e=\Phi^{-1}(-\Phi(x)) .
$$

(iii) It holds that

$$
\left(S_{f} e\right) *\left(S_{g} e\right)=S_{f+g} e \quad \text { and } \quad\left(S_{f} e\right)^{*-1}=S_{-f} e .
$$

Proof. The unit element $e$ is found as $e=S_{-\Phi(y)} y$ with $y \in K_{d}^{+}$being arbitrary. Indeed, the formula $S_{f} x=$ $\Phi^{-1}(f+\Phi(x))$ implied by (A.23) yields

$$
e=\Phi^{-1}(-\Phi(y)+\Phi(y))=\Phi^{-1}(\check{\mathbf{0}})
$$

and hence the above definition of the $*$-multiplication verifies immediately that $x * e=x=e * x$. (This implies also that $S_{-\Phi(y)} y$ is independent of the choice of $y \in K_{d}^{+}$.) Verification of (ii) is straightforward. The assertion (iii) is shown by observing that $S_{f} e=\Phi^{-1}(f+\Phi(e))=\Phi^{-1}(f)$.

Example. Consider the case of $\boldsymbol{\beta}=\mathbf{1}$. Let $x \in K_{d}^{+}$and $f \in \mathbf{R}^{d-1}$. According to Corollary A.1(ii), we have

$$
U(x)=\sum_{i=1}^{d} x_{i} \log x_{i},
$$

and so

$$
\Phi(x)=\nabla U(x)=\left(\log \left(x_{i} / x_{d}\right)\right)_{i=1}^{d-1},
$$

whose inverse admits an explicit form:

$$
\left(\Phi^{-1}(f)\right)_{i}=\frac{e^{f_{i}}}{\sum_{j=1}^{d-1} e^{f_{j}}+1}, \quad i=1, \ldots, d-1 .
$$

Therefore, for each $i \in\{1, \ldots, d-1\}$

$$
\begin{aligned}
\left(S_{f} x\right)_{i}=\left(\Phi^{-1}(f+\Phi(x))\right)_{i} & =\frac{e^{f_{i}+\log \left(x_{i} / x_{d}\right)}}{\sum_{j=1}^{d-1} e^{f_{j}+\log \left(x_{j} / x_{d}\right)}+1} \\
& =\frac{e^{f_{i}} x_{i}}{\sum_{j=1}^{d-1} e^{f_{j}} x_{j}+x_{d}} .
\end{aligned}
$$

Using these expressions, one can easily see that $e=(1 / d, \ldots, 1 / d)^{\prime} \in K_{d}^{+}$and that for each $i \in I$

$$
(x * y)_{i}=\frac{x_{i} y_{i}}{\sum_{j=1}^{d} x_{j} y_{j}} \quad \text { and } \quad\left(x^{*-1}\right)_{i}=\frac{x_{i}{ }^{-1}}{\sum_{j=1}^{d} x_{j}^{-1}} .
$$


Since $\Phi$ is differentiable and its Jacobian matrix (A.22) is nondegenerate, the inverse function theorem implies differentiability (and hence measurability) of mappings $y \mapsto x * y$ and $y \mapsto y^{*-1}$ on $K_{d}^{+}$.

Proposition A.5. Let $F$ be a nonnegative Borel function on $K_{d}^{+}$and take $x \in K_{d}^{+}$arbitrarily. Then it holds that

$$
\int_{\mathbf{R}^{d-1}} F\left(S_{f} x\right) d f_{1} \cdots d f_{d-1}=\int_{K_{d}^{+}} F(y) m(d y)=\int_{K_{d}^{+}} F\left(y^{*-1}\right) m(d y)
$$

and for any $g \in \mathbf{R}^{d-1}$

$$
\int_{K_{d}^{+}} F\left(S_{g} y\right) m(d y)=\int_{K_{d}^{+}} F(y) m(d y)=\int_{K_{d}^{+}} F(x * y) m(d y) .
$$

Proof. The first equality of (A.25) is shown by change of variable $S_{f} x=y$ :

$$
\int_{\mathbf{R}^{d-1}} F\left(S_{f} x\right) d f_{1} \cdots d f_{d-1}=\int_{K_{d}^{+}} F(y) \frac{d y_{1} \cdots d y_{d-1}}{\operatorname{det}\left(D_{f}\left(S_{f} x\right)\right)}=\int_{K_{d}^{+}} F(y) m(d y),
$$

where the last equality uses (S.5). Replacing $F(y)$ by $F\left(y^{*-1}\right)$ and letting $x=e$ (the unit element) in the first equality of (A.25), we get by Lemma A.4(iii)

$$
\begin{aligned}
\int_{K_{d}^{+}} F\left(y^{*-1}\right) m(d y) & =\int_{\mathbf{R}^{d-1}} F\left(\left(S_{f} e\right)^{*-1}\right) d f_{1} \cdots d f_{d-1}=\int_{\mathbf{R}^{d-1}} F\left(S_{-f} e\right) d f_{1} \cdots d f_{d-1} \\
& =\int_{\mathbf{R}^{d-1}} F\left(S_{f} e\right) d f_{1} \cdots d f_{d-1}=\int_{K_{d}^{+}} F(y) m(d y) .
\end{aligned}
$$

Thus the last equality of (A.25) holds. For the proof of the first equality of (A.26), apply (A.25) and (S.2) to show

$$
\begin{aligned}
\int_{K_{d}^{+}} F\left(S_{g} y\right) m(d y) & =\int_{\mathbf{R}^{d-1}} F\left(S_{g}\left(S_{f} e\right)\right) d f_{1} \cdots d f_{d-1}=\int_{\mathbf{R}^{d-1}} F\left(S_{g+f} e\right) d f_{1} \cdots d f_{d-1} \\
& =\int_{\mathbf{R}^{d-1}} F\left(S_{f} e\right) d f_{1} \cdots d f_{d-1}=\int_{K_{d}^{+}} F(y) m(d y) .
\end{aligned}
$$

This implies also the other equality in (A.26). Indeed, taking $f \in \mathbf{R}^{d-1}$ such that $x=S_{f} e$, we have $x * y=$ $\Phi^{-1}(f+\Phi(y))=S_{f} y$ and hence

$$
\int_{K_{d}^{+}} F(x * y) m(d y)=\int_{K_{d}^{+}} F\left(S_{f} y\right) m(d y)=\int_{K_{d}^{+}} F(y) m(d y) .
$$

The proof of Proposition A.5 is completed.

According to general theory of Haar measures (see e.g. [9]), we have uniqueness (up to multiplicative constants) of regular measures on $K_{d}^{+}$which are invariant under $\left\{S_{f}\right\}$. In fact, as we will see below, the uniqueness is easily 
shown by using the first equality in (A.25). Suppose that a regular Borel measure $n(d x)$ on $K_{d}^{+}$is invariant under $\left\{S_{f}\right\}$. Then for arbitrary nonnegative Borel functions $F(x)$ and $G(x)$ on $K_{d}^{+}$,

$$
\int_{K_{d}^{+}} F\left(S_{f} x\right) G(x) n(d x)=\int_{K_{d}^{+}} F(x) G\left(S_{-f} x\right) n(d x) .
$$

Integrating both sides with respect to $d f_{1} \cdots d f_{d-1}$ over $\mathbf{R}^{d-1}$, we have by Fubini's theorem and (A.25)

$$
\int_{K_{d}^{+}} F(y) m(d y) \int_{K_{d}^{+}} G(x) n(d x)=\int_{K_{d}^{+}} F(x) n(d x) \int_{K_{d}^{+}} G(y) m(d y) .
$$

This implies that there exists a nonnegative finite constant $c$ such that $n(E)=c m(E)$ for every Borel subset $E$ of $K_{d}^{+}$. See the proof of Theorem C $(\S 60)$ in [9] for more details.

\section{References}

[1] R. Bürger, The Mathematical Theory of Selection, Recombination, and Mutation, Wiley, Chichester, 2000.

[2] D.M. Cifarelli, E. Regazzini, Distribution functions of means of a Dirichlet process, Ann. Statist. 18 (1990) 429-442. Correction: Ann. Statist. 22 (1994) 1633-1634.

[3] S.N. Ethier, T.G. Kurtz, Markov Processes: Characterization and Convergence, Wiley, New York, 1986.

[4] S.N. Ethier, T.G. Kurtz, Fleming-Viot processes in population genetics, SIAM J. Contr. Optim. 31 (1993) 345-386.

[5] W.J. Ewens, Mathematical Population Genetics, Springer, Berlin, 1979.

[6] M. Fukushima, D. Stroock, Reversibility of solutions to martingale problems, in: Adv. Math. Suppl. Stud., vol. 9, Academic Press, Orlando, 1986, pp. 107-123.

[7] J.H. Gillespie, Natural selection for within-generation variance in offspring number, Genetics 76 (1974) 601-606.

[8] A. Guionnet, B. Zegarlinski, Lectures on logarithmic Sobolev inequalities, in: Séminaire de Probabilités XXXVI, in: Lecture Notes in Math., vol. 1801, Springer, Berlin, 2003, pp. 1-134.

[9] P.R. Halmos, Measure Theory, in: Graduate Texts in Mathematics, vol. 18, Springer, New York, 1974.

[10] K. Handa, Quasi-invariant measures and their characterization by conditional probabilities, Bull. Sci. Math. 125 (2001) $583-604$.

[11] K. Handa, Quasi-invariance and reversibility in the Fleming-Viot process, Probab. Theory Related Fields 122 (2002) 545-566.

[12] R. Holley, D. Stroock, Simulated annealing via Sobolev inequalities, Comm. Math. Phys. 115 (1988) 553-569.

[13] N. Ikeda, S. Watanabe, Stochastic Differential Equations and Diffusion Processes, second ed., North-Holland/Kodansha, Amsterdam/Tokyo, 1989.

[14] A.N. Kolmogoroff, Zur Umkehrbarkeit der statistischen Naturgesetze, Math. Ann. 113 (1937) 766-772.

[15] B. Levikson, The age distribution of Markov processes, J. Appl. Probab. 14 (1977) 492-506.

[16] Z. Li, T. Shiga, L. Yao, A reversibility problem for Fleming-Viot processes, Elect. Comm. Probab. 4 (1999) 71-82.

[17] M. Nagasawa, T. Maruyama, An application of time reversal of Markov processes to a problem of population genetics, Adv. Appl. Probab. 11 (1979) 457-478.

[18] E. Nelson, The adjoint Markoff process, Duke Math. J. 25 (1958) 671-690.

[19] K. Sato, Convergence to a diffusion of a multi-allelic model in population genetics, Adv. Appl. Probab. 10 (1978) 538-562.

[20] S. Sawyer, On the past history of an allele now known to have frequency p, J. Appl. Probab. 14 (1977) $439-450$.

[21] T. Shiga, Diffusion processes in population genetics, J. Math. Kyoto Univ. 21 (1981) 133-151.

[22] T. Shiga, A certain class of infinite-dimensional diffusion processes arising in population genetics, J. Math. Soc. Japan 39 (1987) 17-25.

[23] T. Shiga, Multi-allelic Gillespie-Sato diffusion models and their extension to infinite allelic ones, in: M. Kimura, G. Kallianpur, T. Hida (Eds.), Stochastic Methods in Biology (Nagoya, 1985), in: Lecture Notes in Biomath., vol. 70, Springer, Berlin, 1987, pp. 87-99.

[24] T. Shiga, A stochastic equation based on a Poisson system for a class of measure-valued diffusion processes, J. Math. Kyoto Univ. 30 (1990) 245-279.

[25] W. Stannat, On the validity of the log-Sobolev inequality for symmetric Fleming-Viot operators, Ann. Probab. 28 (2000) 667-684.

[26] N. Tsilevich, A. Vershik, M. Yor, Distinguished properties of the gamma process, and related topics, Prépublication du Laboratoire de Probabilités et Moèles Aléatoires No. 575, 2000, available at http://xxx.lanl.gov/ps/math.PR/0005287.

[27] N. Tsilevich, A. Vershik, M. Yor, An infinite-dimensional analogue of the Lebesgue measure and distinguished properties of the gamma process, J. Funct. Anal. 185 (2001) 274-296.

[28] G.A. Watterson, Reversibility and the age of an allele, I, Moran's infinitely many neutral alleles model, Theoret. Population Biology 10 (1976) 239-253. 
[29] G.A. Watterson, Reversibility and the age of an allele, II, Two-allele models, with selection and mutation, Theoret. Population Biology 12 (1977) 179-196.

[30] S. Wright, Adaptation and selection, in: G.L. Jepson, E. Mayr, G.G. Simpson (Eds.), Genetics, Paleontology, and Evolution, Princeton University, Princeton, NJ, 1949, pp. 365-389. 